\title{
Search for non-relativistic magnetic monopoles with IceCube
}

\author{
IceCube Collaboration
}

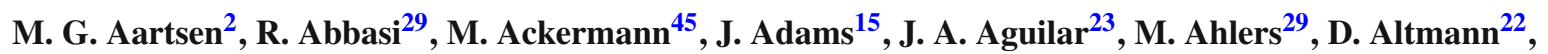
C. Arguelles ${ }^{29}$, T. C. Arlen ${ }^{42}$, J. Auffenberg ${ }^{1}$, X. Bai ${ }^{33, c}$, M. Baker ${ }^{29}$, S. W. Barwick ${ }^{25}$, V. Baum ${ }^{30}$, R. Bay ${ }^{7}$, J. J. Beatty ${ }^{17,18}$, J. Becker Tjus ${ }^{10}$, K.-H. Becker ${ }^{44}$, M. L. Benabderrahmane ${ }^{45, b}$, S. BenZvi ${ }^{29}$, P. Berghaus ${ }^{45}$, D. Berley ${ }^{16}$, E. Bernardini ${ }^{45}$, A. Bernhard ${ }^{32}$, D. Z. Besson ${ }^{27}$, G. Binder ${ }^{7,8}$, D. Bindig ${ }^{44}$, M. Bissok ${ }^{1}$, E. Blaufuss ${ }^{16}$, J. Blumenthal ${ }^{1}$, D. J. Boersma ${ }^{43}$, C. Bohm ${ }^{36}$, D. Bose ${ }^{38}$, S. Böser ${ }^{11}$, O. Botner ${ }^{43}$, L. Brayeur ${ }^{13}$, H.-P. Bretz ${ }^{45}$, A. M. Brown ${ }^{15}$, R. Bruijn ${ }^{26}$, J. Casey ${ }^{5}$, M. Casier ${ }^{13}$, D. Chirkin ${ }^{29}$, A. Christov ${ }^{23}$, B. Christy ${ }^{16}$, K. Clark ${ }^{39}$, L. Classen ${ }^{22}$, F. Clevermann ${ }^{20}$, S. Coenders ${ }^{32}$, S. Cohen ${ }^{26}$, D. F. Cowen ${ }^{41,42}$, A. H. Cruz Silva ${ }^{45}$, M. Danninger ${ }^{36}$, J. Daughhetee ${ }^{5}$, J. C. Davis ${ }^{17}$, M. Day ${ }^{29}$, J. P. A. M. de André ${ }^{42}$, C. De Clercq $^{13}$, S. De Ridder ${ }^{24}$, P. Desiati ${ }^{29}$, K. D. de Vries $^{13}$, M. de With ${ }^{9}$, T. DeYoung ${ }^{42}$, J. C. Díaz-Vélez ${ }^{29}$, M. Dunkman ${ }^{42}$, R. Eagan ${ }^{42}$, B. Eberhardt ${ }^{30}$, B. Eichmann ${ }^{10}$, J. Eisch $^{29}$, S. Euler ${ }^{43}$, P. A. Evenson ${ }^{33}$, O. Fadiran ${ }^{29}$, A. R. Fazely ${ }^{6}$, A. Fedynitch ${ }^{10}$, J. Feintzeig ${ }^{29}$, T. Feusels ${ }^{24}$, K. Filimonov ${ }^{7}$, C. Finley ${ }^{36}$, T. Fischer-Wasels ${ }^{44}$, S. Flis ${ }^{36}$, A. Franckowiak ${ }^{11}$, K. Frantzen ${ }^{20}$, T. Fuchs ${ }^{20}$, T. K. Gaisser ${ }^{33}$, J. Gallagher ${ }^{28}$, L. Gerhardt ${ }^{7,8}$, L. Gladstone ${ }^{29}$, T. Glüsenkamp ${ }^{45}$, A. Goldschmidt ${ }^{8}$, G. Golup ${ }^{13}$, J. G. Gonzalez ${ }^{33}$, J. A. Goodman ${ }^{16}$, D. Góra ${ }^{22}$, D. T. Grandmont ${ }^{21}$, D. Grant ${ }^{21}$, P. Gretskov ${ }^{1}$, J. C. Groh ${ }^{42}$, A. Groß ${ }^{32}$, C. Ha ${ }^{7,8}$, C. Haack ${ }^{1}$, A. Haj Ismail ${ }^{24}$, P. Hallen ${ }^{1}$, A. Hallgren ${ }^{43}$, F. Halzen ${ }^{29}$, K. Hanson ${ }^{12}$, D. Hebecker ${ }^{11}$, D. Heereman ${ }^{12}$, D. Heinen ${ }^{1}$, K. Helbing ${ }^{44}$, R. Hellauer ${ }^{16}$, S. Hickford ${ }^{15}$, G. C. Hill ${ }^{2}$, K. D. Hoffman ${ }^{16}$, R. Hoffmann ${ }^{44}$, A. Homeier ${ }^{11}$, K. Hoshina ${ }^{29, d}$, F. Huang ${ }^{42}$, W. Huelsnitz ${ }^{16}$, P. O. Hulth ${ }^{36}$, K. Hultqvist ${ }^{36}$, S. Hussain ${ }^{33}$, A. Ishihara ${ }^{14}$, E. Jacobi ${ }^{45}$, J. Jacobsen ${ }^{29}$, K. Jagielski ${ }^{1}$, G. S. Japaridze ${ }^{4}$, K. Jero ${ }^{29}$, O. Jlelati ${ }^{24}$, B. Kaminsky ${ }^{45}$, A. Kappes ${ }^{22}$, T. Karg ${ }^{45}$, A. Karle ${ }^{29}$, M. Kauer ${ }^{29}$, J. L. Kelley ${ }^{29}$, J. Kiryluk ${ }^{37}$, J. Kläs ${ }^{44}$, S. R. Klein ${ }^{7,8}$, J.-H. Köhne ${ }^{20}$, G. Kohnen ${ }^{31}$, H. Kolanoski ${ }^{9}$, L. Köpke ${ }^{30}$, C. Kopper ${ }^{29}$, S. Kopper ${ }^{44}$, D. J. Koskinen ${ }^{19}$, M. Kowalski ${ }^{11}$, M. Krasberg ${ }^{29}$, A. Kriesten ${ }^{1}$, K. Krings ${ }^{1}$, G. Kroll ${ }^{30}$, J. Kunnen ${ }^{13}$, N. Kurahashi ${ }^{29}$, T. Kuwabara ${ }^{33}$, M. Labare ${ }^{24}$, H. Landsman ${ }^{29}$, M. J. Larson ${ }^{40}$, M. Lesiak-Bzdak ${ }^{37}$, M. Leuermann ${ }^{1}$, J. Leute ${ }^{32}$, J. Lünemann ${ }^{30}$, O. Macías ${ }^{15}$,

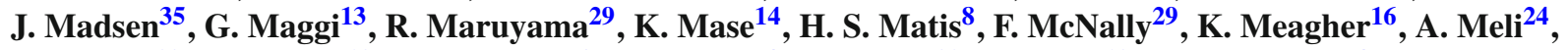
M. Merck $^{29}$, T. Meures ${ }^{12}$, S. Miarecki ${ }^{7,8}$, E. Middell ${ }^{45}$, N. Milke ${ }^{20}$, J. Miller ${ }^{13}$, L. Mohrmann ${ }^{45}$, T. Montaruli ${ }^{23}$, R. Morse $^{29}$, R. Nahnhauer ${ }^{45}$, U. Naumann ${ }^{44}$, H. Niederhausen ${ }^{37}$, S. C. Nowicki ${ }^{21}$, D. R. Nygren ${ }^{8}$, A. Obertacke ${ }^{44}$, S. Odrowski ${ }^{21}$, A. Olivas ${ }^{16}$, A. Omairat ${ }^{44}$, A. O'Murchadha ${ }^{12}$, T. Palczewski ${ }^{40}$, L. Paul ${ }^{1}$, J. A. Pepper ${ }^{40}$, C. Pérez de $\operatorname{los}_{\operatorname{Heros}^{43}}$, C. Pfendner ${ }^{17}$, D. Pieloth ${ }^{20}$, E. Pinat ${ }^{12}$, J. Posselt ${ }^{44}$, P. B. Price ${ }^{7}$, G. T. Przybylski ${ }^{8}$, M. Quinnan ${ }^{42}$, L. Rädel ${ }^{1}$, M. Rameez ${ }^{23}$, K. Rawlins ${ }^{3}$, P. Redl ${ }^{16}$, R. Reimann ${ }^{1}$, E. Resconi $^{32}$, W. Rhode $^{20}$, M. Ribordy ${ }^{26}$, M. Richman ${ }^{16}$, B. Riedel ${ }^{29}$, S. Robertson ${ }^{2}$, J. P. Rodrigues ${ }^{29}$, C. Rott $^{38}$, T. Ruhe ${ }^{20}$, B. Ruzybayev ${ }^{33}$, D. Ryckbosch ${ }^{24}$, S. M. Saba ${ }^{10}$, H.-G. Sander ${ }^{30}$, M. Santander ${ }^{29}$, S. Sarkar ${ }^{19,34}$, K. Schatto ${ }^{30}$, F. Scheriau ${ }^{20}$, T. Schmidt ${ }^{16}$, M. Schmitz ${ }^{20}$, S. Schoenen ${ }^{1, a}$, S. Schöneberg ${ }^{10}$, A. Schönwald ${ }^{45}$, A. Schukraft ${ }^{1}$, L. Schulte ${ }^{11}$, O. Schulz $^{32}$, D. Seckel ${ }^{33}$, Y. Sestayo ${ }^{32}$, S. Seunarine ${ }^{35}$, R. Shanidze ${ }^{45}$, C. Sheremata ${ }^{21}$, M. W. E. Smith ${ }^{42}$, D. Soldin ${ }^{44}$, G. M. Spiczak ${ }^{35}$, C. Spiering ${ }^{45}$, M. Stamatikos ${ }^{17, e}$, T. Stanev ${ }^{33}$, N. A. Stanisha ${ }^{42}$, A. Stasik ${ }^{11}$, T. Stezelberger ${ }^{8}$, R. G. Stokstad ${ }^{8}$, A. Stößl ${ }^{45}$, E. A. Strahler ${ }^{13}$, R. Ström ${ }^{43}$, N. L. Strotjohann ${ }^{11}$, G. W. Sullivan ${ }^{16}$, H. Taavola ${ }^{43}$,

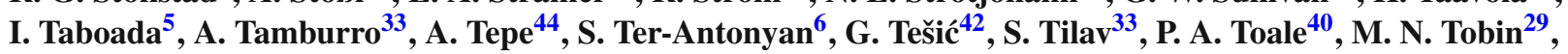
S. Toscano ${ }^{29}$, M. Tselengidou ${ }^{22}$, E. Unger ${ }^{10}$, M. Usner ${ }^{11}$, S. Vallecorsa ${ }^{23}$, N. van Eijndhoven $^{13}$, J. van Santen ${ }^{29}$, M. Vehring ${ }^{1}$, M. Voge ${ }^{11}$, M. Vraeghe ${ }^{24}$, C. Walck ${ }^{36}$, M. Wallraff ${ }^{1}$, Ch. Weaver ${ }^{29}$, M. Wellons ${ }^{29}$, C. Wendt ${ }^{29}$, S. Westerhoff ${ }^{29}$, B. J. Whelan ${ }^{2}$, N. Whitehorn ${ }^{29}$, K. Wiebe $^{30}$, C. H. Wiebusch ${ }^{1}$, D. R. Williams ${ }^{40}$, H. Wissing ${ }^{16}$, M. Wolf ${ }^{36}$, T. R. Wood ${ }^{21}$, K. Woschnagg ${ }^{7}$, D. L. Xu ${ }^{40}$, X. W. Xu' ${ }^{6}$, J. P. Yanez ${ }^{45}$, G. Yodh ${ }^{25}$, S. Yoshida ${ }^{14}$, P. Zarzhitsky ${ }^{40}$, J. Ziemann ${ }^{20}$, S. Zierke ${ }^{1}$, M. Zoll ${ }^{36}$

\footnotetext{
${ }^{1}$ III. Physikalisches Institut, RWTH Aachen University, 52056 Aachen, Germany

2 School of Chemistry and Physics, University of Adelaide, Adelaide, SA 5005, Australia

${ }^{3}$ Department of Physics and Astronomy, University of Alaska Anchorage, 3211 Providence Dr., Anchorage, AK 99508, USA

${ }^{4}$ CTSPS, Clark-Atlanta University, Atlanta, GA 30314, USA

${ }^{5}$ School of civistic Astrophysics, Georgia Institute of Technology, Atlanta, GA 30332, USA

${ }^{6}$ Department of Physics, Southern University, Baton Rouge, LA 70813, USA
} 
${ }^{7}$ Department of Physics, University of California, Berkeley, CA 94720, USA

${ }^{8}$ Lawrence Berkeley National Laboratory, Berkeley, CA 94720, USA

${ }^{9}$ Institut für Physik, Humboldt-Universität zu Berlin, 12489 Berlin, Germany

${ }^{10}$ Fakultät für Physik \& Astronomie, Ruhr-Universität Bochum, 44780 Bochum, Germany

${ }^{11}$ Physikalisches Institut, Universität Bonn, Nussallee 12, 53115 Bonn, Germany

12 Université Libre de Bruxelles, Science Faculty CP230, 1050 Brussels, Belgium

13 Vrije Universiteit Brussel, Dienst ELEM, 1050 Brussels, Belgium

${ }^{14}$ Department of Physics, Chiba University, Chiba 263-8522, Japan

15 Department of Physics and Astronomy, University of Canterbury, Private Bag 4800, Christchurch, New Zealand

${ }^{16}$ Department of Physics, University of Maryland, College Park, MD 20742, USA

${ }^{17}$ Department of Physics and Center for Cosmology and Astro-Particle Physics, Ohio State University, Columbus, OH 43210, USA

18 Department of Astronomy, Ohio State University, Columbus, OH 43210, USA

${ }^{19}$ Niels Bohr Institute, University of Copenhagen, 2100 Copenhagen, Denmark

${ }^{20}$ Department of Physics, TU Dortmund University, 44221 Dortmund, Germany

${ }^{21}$ Department of Physics, University of Alberta, Edmonton, AB T6G 2E1, Canada

${ }^{22}$ Erlangen Centre for Astroparticle Physics, Friedrich-Alexander-Universität Erlangen-Nürnberg, 91058 Erlangen, Germany

${ }^{23}$ Département de physique nucléaire et corpusculaire, Université de Genève, 1211 Geneva, Switzerland

${ }^{24}$ Department of Physics and Astronomy, University of Gent, 9000 Gent, Belgium

${ }^{25}$ Department of Physics and Astronomy, University of California, Irvine, CA 92697, USA

${ }^{26}$ Laboratory for High Energy Physics, École Polytechnique Fédérale, 1015 Lausanne, Switzerland

${ }^{27}$ Department of Physics and Astronomy, University of Kansas, Lawrence, KS 66045, USA

28 Department of Astronomy, University of Wisconsin, Madison, WI 53706, USA

${ }^{29}$ Department of Physics and Wisconsin IceCube Particle Astrophysics Center, University of Wisconsin, Madison, WI 53706, USA

${ }^{30}$ Institute of Physics, University of Mainz, Staudinger Weg 7, 55099 Mainz, Germany

${ }^{31}$ Université de Mons, 7000 Mons, Belgium

32 T.U. Munich, 85748 Garching, Germany

${ }^{33}$ Bartol Research Institute and Department of Physics and Astronomy, University of Delaware, Newark, DE 19716, USA

${ }^{34}$ Department of Physics, University of Oxford, 1 Keble Road, Oxford OX1 3NP, UK

35 Department of Physics, University of Wisconsin, River Falls, WI 54022, USA

${ }^{36}$ Oskar Klein Centre and Department of Physics, Stockholm University, 10691 Stockholm, Sweden

${ }^{37}$ Department of Physics and Astronomy, Stony Brook University, Stony Brook, NY 11794-3800, USA

${ }^{38}$ Department of Physics, Sungkyunkwan University, Suwon 440-746, Korea

39 Department of Physics, University of Toronto, Toronto, ON M5S 1A7, Canada

${ }^{40}$ Department of Physics and Astronomy, University of Alabama, Tuscaloosa, AL 35487, USA

${ }^{41}$ Department of Astronomy and Astrophysics, Pennsylvania State University, University Park, PA 16802, USA

42 Department of Physics, Pennsylvania State University, University Park, PA 16802, USA

43 Department of Physics and Astronomy, Uppsala University, Box 516, 75120 Uppsala, Sweden

44 Department of Physics, University of Wuppertal, 42119 Wuppertal, Germany

45 DESY, 15735 Zeuthen, Germany

Received: 14 February 2014 / Accepted: 11 June 2014 / Published online: 2 July 2014 (C) The Author(s) 2014. This article is published with open access at Springerlink.com

Abstract The IceCube Neutrino Observatory is a large Cherenkov detector instrumenting $1 \mathrm{~km}^{3}$ of Antarctic ice. The detector can be used to search for signatures of particle physics beyond the Standard Model. Here, we describe the search for non-relativistic, magnetic monopoles as remnants of the Grand $U$ nified $T$ heory (GUT) era shortly after the Big Bang. Depending on the underlying gauge group these monopoles may catalyze the decay of nucleons via

\footnotetext{
a e-mail: schoenen@physik.rwth-aachen.de

b e-mail: mohamed.lotfi.benabderrahmane@desy.de

${ }^{c}$ Physics Department, South Dakota School of Mines and Technology, Rapid City, SD 57701, USA

d Earthquake Research Institute, University of Tokyo, Bunkyo,

Tokyo 113-0032, Japan

e NASA Goddard Space Flight Center, Greenbelt, MD 20771, USA
}

the Rubakov-Callan effect with a cross section suggested to be in the range of $10^{-27}$ to $10^{-21} \mathrm{~cm}^{2}$. In IceCube, the Cherenkov light from nucleon decays along the monopole trajectory would produce a characteristic hit pattern. This paper presents the results of an analysis of first data taken from May 2011 until May 2012 with a dedicated slowparticle trigger for DeepCore, a subdetector of IceCube. A second analysis provides better sensitivity for the brightest non-relativistic monopoles using data taken from May 2009 until May 2010. In both analyses no monopole signal was observed. For catalysis cross sections of $10^{-22}\left(10^{-24}\right) \mathrm{cm}^{2}$ the flux of non-relativistic GUT monopoles is constrained up to a level of $\Phi_{90} \leq 10^{-18}\left(10^{-17}\right) \mathrm{cm}^{-2} \mathrm{~s}^{-1} \mathrm{sr}^{-1}$ at a $90 \%$ confidence level, which is three orders of magnitude below the Parker bound. The limits assume a dominant decay of the proton into a positron and a neutral pion. These results 
improve the current best experimental limits by one to two orders of magnitude, for a wide range of assumed speeds and catalysis cross sections.

\section{Introduction}

Magnetic monopoles are particles carrying a quantized magnetic charge and are predicted in various theories. In classical electrodynamics, their existence would symmetrize Maxwell's equations with respect to the sources of the electromagnetic field. Quantum mechanically, the existence of magnetic monopoles implies that both electric charge and the hypothetical magnetic charge, are quantized, given that the associated electromagnetic fields still satisfy Maxwell's equations [1]. The resulting magnetic elementary charge, called the Dirac charge $g_{\mathrm{D}}$, is

$g_{\mathrm{D}}=\frac{e}{2 \alpha}$,

where $e$ is the electric elementary charge and $\alpha$ is the fine structure constant.

In Grand Unified Theories (GUTs) [2] magnetic monopoles appear as stable, finite-energy solutions of the field equations $[3,4]$. The predicted masses range from $10^{5}$ to $10^{17} \mathrm{GeV}$ [5-9] and their magnetic charges are integer multiples of the Dirac charge $g_{\mathrm{D}}$. The lower part of the mass range up to $\sim 10^{13} \mathrm{GeV}$ refers to intermediate mass monopoles (IMMs) which arise from intermediate stages of symmetry breaking below the GUT scale. In contrast the superheavy monopoles with masses at the GUT scale may have been created during the phase transition associated with the spontaneous breakdown of the unified gauge symmetry in the early universe at $\sim 10^{-36} \mathrm{~s}$ after the Big Bang [10]. The monopole mass and charge depend on the underlying gauge group, the symmetry breaking hierarchy, and the type and temperature of the phase transition in a particular GUT.

Since magnetic monopoles are stable, they should still be present in cosmic rays. The number density today depends on the existence of an inflationary epoch and on the time of creation, which could be before, during or after this epoch [11]. Since then, monopoles have been accelerated by large-scale cosmic magnetic fields. The kinetic-energy gain by passing through a magnetic field $B$ is given by

$E_{\text {kin }}=g \int_{\text {path }} \mathbf{B} \cdot \mathrm{d} \mathbf{l}$,

where $g=n \cdot g_{\mathrm{D}}$ is the magnetic charge. The maximum kinetic energy of a magnetic monopole due to acceleration in cosmic magnetic fields is rather uncertain but can reach $\sim 10^{14} \mathrm{GeV}$ [9]. Therefore, monopoles with masses at, or above, this energy scale should be non-relativistic. Based on the propagation of magnetic monopoles in the Galactic magnetic field an upper bound on the monopole flux can be calculated, assuming the Galactic magnetic field does not decrease faster than it can be regenerated. This assumption constrains the monopole flux to be less than $10^{-15} \mathrm{~cm}^{-2} \mathrm{~s}^{-1} \mathrm{sr}^{-1}$, which is called the Parker Bound [12,13]. Taking into account the fields during galaxy formation, the limit was extended by Adams et al. to be less than $10^{-16} \mathrm{~cm}^{-2} \mathrm{~s}^{-1} \mathrm{sr}^{-1}$ for monopoles with masses below $10^{17} \mathrm{GeV}$ [14].

Many experiments have searched for relic magnetic monopoles, but there is no experimental proof for their existence. The current best limits for magnetic monopoles constrain their flux to a level of $\sim 10^{-16}-10^{-18} \mathrm{~cm}^{-2} \mathrm{~s}^{-1} \mathrm{sr}^{-1}$ depending on the monopole speed and interaction mechanism [15-18]. Consequently, searches for magnetic monopoles require very large detectors.

The IceCube Neutrino Observatory currently is the world's largest neutrino detector. The primary goal is the detection of Cherenkov light from electrically charged secondary particles produced when high-energy astrophysical neutrinos interact in the surrounding matter [19]. However, IceCube can also be used to search for magnetic monopoles. Depending on their speed monopoles have different signatures in IceCube. Relativistic monopoles with a speed above the Cherenkov threshold, e.g. $\beta \approx 0.76$ in ice, can be detected by the Cherenkov light they directly produce [20]. Non-relativistic monopoles that catalyze the decay of nucleons in the detector medium can, in contrast, be detected by the Cherenkov light from electrically charged secondary particles produced in subsequent nucleon decays along the monopole trajectory (Sect. 2.2). Therefore, different analysis strategies are needed in order to cover both detection channels. This paper presents the results of a search for non-relativistic magnetic monopoles which would catalyze the proton decays via the Rubakov-Callan effect in IceCube.

\section{Monopole detection with IceCube}

\subsection{The IceCube detector}

The IceCube Neutrino Observatory consists of the in-ice detector, IceCube, and the surface air shower detector, IceTop. It is located at the geographic South Pole. For the in-ice detector, $1 \mathrm{~km}^{3}$ of the Antarctic ice, which is used as detection medium, has been instrumented. The detector consists of 86 strings equipped with 60 digital optical modules (DOMs) each. The DOM, the sensor of the IceCube detector, consists of a glass pressure housing enclosing a $25.4 \mathrm{~cm}$ diameter Hamamatsu photomultiplier tube (PMT) with the electronics needed for signal digitization, and a set of LEDs for calibration purposes [21,22]. Signals that pass a threshold of about 
0.25 photo-electrons are digitized and recorded. This process is called a DOM launch or for simplicity a hit in the following. Two hits are labeled as hard local coincidences (also called HLC pair), if their time difference is less than $1 \mu \mathrm{s}$ and the corresponding DOMs are nearest or next-to-nearest neighbors on the same string. The recorded data is sent to the surface and a trigger algorithm evaluates the time and position of the hits and decides whether they form an event. For example, for relativistic particles a simple multiplicity trigger requiring eight HLC hits within a time window of $5 \mu \mathrm{s}$, called SMT8, is used. The DOMs are deployed at depths between 1450 and $2450 \mathrm{~m}$ [23]. At depths below $2100 \mathrm{~m}$, eight inner strings are placed with smaller separations from each other and thus form a region of denser instrumentation. Together with seven central standard strings they form DeepCore, a low-energy subdetector [24]. The construction of IceCube was completed December 16, 2010 but data taken during intermediate construction stages were already used for physics analyses during earlier years. One of the two presented analyses uses data taken from May 2009 to May 2010, when IceCube was operating in its 59-string configuration (IC-59). The other analysis uses the fully installed detector.

\subsection{The Rubakov-Callan effect}

Non-relativistic magnetic monopoles would themselves be too slow to emit Cherenkov light when propagating through the IceCube detector. However, relativistic charged secondary particles, produced in monopole interactions with the surrounding matter, can produce Cherenkov light and thus can be detected by the IceCube detector.

The energy loss of a magnetic monopole due to ionization can be described by a modified Bethe-Bloch formula [25-27], which is valid for speeds $\beta>0.1$. For lower speeds in the range from $\beta=10^{-3}$ to $10^{-2}$ Ahlen and Kinoshita introduced a model to calculate the energy loss of magnetic monopoles [28]. Later, Ritson extended this model for speeds below $\beta=10^{-3}$ [29]. For magnetic monopoles with e.g. $\beta=10^{-3}$ the energy loss is of the order of $20 \mathrm{MeV} \mathrm{g}^{-1} \mathrm{~cm}^{2}$ [13]. Only electrons above the Cherenkov threshold of $\sim 0.28 \mathrm{MeV}$ kinetic energy emit detectable Cherenkov light. However, the maximum transferred energy of a monopole with e.g. $\beta=10^{-3}$ on an atomic electron is typically $E_{\max } \simeq 10 \mathrm{eV}$ and no Cherenkov light is produced.

Rubakov [30] and Callan [31] showed that some monopoles could catalyze nucleon decays along their trajectories (Rubakov-Callan effect). This effect depends on the gauge group of the respective GUT theory $[32,33]$ and on assumptions, e.g. on the fermion masses or the relative velocity between the quarks and the monopole, used in the calculation $[34,35]$. Furthermore, this process is not possible for intermediate mass monopoles with masses below $\sim 10^{13} \mathrm{GeV}$ [8].

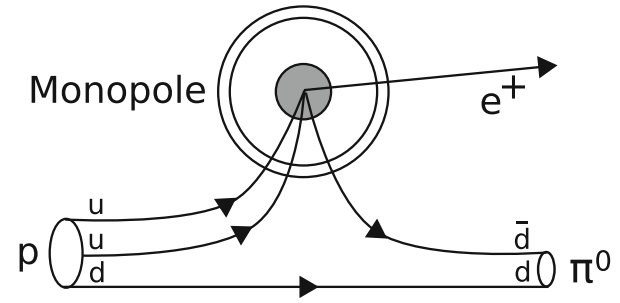

Fig. 1 Illustration of a proton decay into a positron and a neutral pion catalyzed by a GUT monopole

Therefore, the sensitivity of this analysis is contrained to heavier monopoles (GUT scale). Figure 1 illustrates the catalyzed decay of a proton by a GUT monopole into a positron and a neutral pion:

$M+p \rightarrow M+\mathrm{e}^{+}+\pi^{0}$.

For this decay channel almost the full rest mass energy of the proton is transferred to electromagnetic particles. Because of the high light yield this channel is used as a benchmark in the analyses.

The catalysis cross section for nucleon decays $\sigma_{\text {cat }}$ depends not only on the cross section $\sigma_{0}$ [36], but also on the monopole speed $\beta=v / c$ :

$\sigma_{\text {cat }}= \begin{cases}\frac{\sigma_{0}}{\beta} & \text { for } \beta \geq \beta_{0} \\ \frac{\sigma_{0}}{\beta} \cdot F(\beta) & \text { for } \beta<\beta_{0} .\end{cases}$

The correction $F(\beta)=\left(\frac{\beta}{\beta_{0}}\right)^{\gamma}$ takes into account an additional angular momentum of the monopole-nucleus-system and becomes relevant for speeds below the speed threshold $\beta_{0}$. Depending on the sign of $\gamma$ the catalysis cross section is enhanced or suppressed. Both parameters $\gamma$ and $\beta_{0}$ depend on the nucleus [37]. Current estimates for the catalysis cross sections are of the order of $10^{-27} \mathrm{~cm}^{2}$ to $10^{-21} \mathrm{~cm}^{2}$ [38].

The Rubakov-Callan effect results in small electromagnetic or hadronic cascades from catalyzed nucleon decays along the monopole trajetory through the detector. This is illustrated in Fig. 2. Experimentally, the relevant parameter is the mean free path $\lambda_{\text {cat }}$ between two decays. That is

$\lambda_{\text {cat }}=\frac{1}{\sigma_{\text {cat }} \cdot n}$,

where $n$ is the particle density of the medium through which the monopole propagates.

The energy of each cascade, and therefore the number of emitted Cherenkov photons, depends on the decay channel (e.g. Eq. 3). A general quantity is the track length, $l_{\gamma}$, the distance a relativistic particle carrying a single electric charge would have to travel in order to emit the same number of Cherenkov photons as the average number expected from a proton decay, $N_{\gamma}$ [39]. Using this track length per proton decay, $l_{\gamma}$, the monopole's mean free path $\lambda_{\text {cat }}$ can 


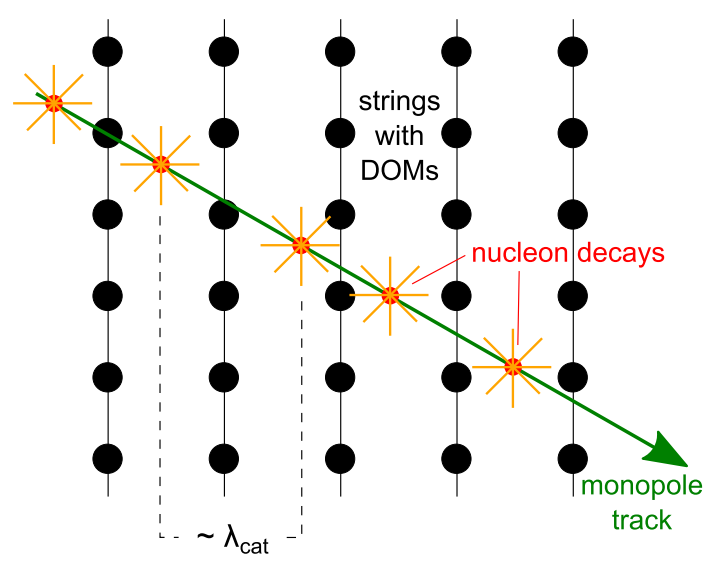

Fig. 2 Illustration of the signature of a non-relativistic magnetic monopole (green) catalyzing nucleon decays (red) along its track in IceCube. The resulting cascades with mean distances $\lambda_{\text {cat }}$ are symbolized by orange rays

be converted into the light yield per monopole track length $\hat{l}$.

$\hat{l}=\frac{l_{\gamma}}{\lambda_{\text {cat }}} \propto \frac{N_{\gamma}}{\lambda_{\text {cat }}}$.

A monopole with $\hat{l}=1$ will therefore produce the same number of Cherenkov photons per track length as a singleelectric-charge, relativistic particle without stochastic energy losses along its track [40]. This implies that $\hat{l}$ can be used to express the resulting monopole flux limits without assuming a specific decay channel (Sect. 6). This ansatz is valid as long as the monopole's light emission can be approximated as being continuous. This condition is satisfied for a mean free path much smaller than the detector spacing. From an experimental point of view the speed $\beta$ and the mean free path $\lambda_{\text {cat }}$ are the characterizing parameters for the detection of such monopoles.

Searches for slow monopoles based on the RubakovCallan effect have been pioneered with the underground detectors IMB and Kamiokande-II [41,42] and the underwater detectors in Lake Baikal [43-45]. A similar search has also been performed with AMANDA, the predecessor of IceCube [46].

During the commissioning of the full detector (IC-86) in May 2011, a dedicated trigger for slow particle signatures (Slow-Particle Trigger, Sect. 3.1) in DeepCore was implemented. The denser instrumentation of DeepCore allows IceCube to detect monopoles of low light emission, i.e. with rather large values of mean distances $\lambda_{\text {cat }}$ between induced catalysis points. In 2009, the deployment of the first DeepCore strings was still ahead. Due to the larger spacing and the lack of an appropriate trigger, IC-59 was blind for large $\lambda_{\text {cat }}$. For smaller $\lambda_{\text {cat }}$ the mentioned drawbacks were balanced by the larger geometrical area compared to DeepCore.

\subsection{Simulation of magnetic monopoles}

The signal expectation was determined from Monte Carlo simulations of magnetic monopoles in IceCube, while the background expectation was determined from experimental data itself, with only supplementary simulations.

IceCube simulation includes particle injection and propagation, taking into account appropriate particle interactions, as well as the full detector response to the generated Cherenkov photons.

The arrival directions of magnetic monopoles are assumed to be isotropic. The starting points of simulated monopole tracks are generated randomly on a disc of fixed size. The distance of the plane is fixed with respect to the DeepCore detector but its orientation is random. It is assumed that the magnetic monopoles are not substantially decelerated along their track and their velocity is constant [47].

The distances between the catalyzed nucleon decays are simulated as a Poisson process with a mean free path $\lambda_{\text {cat }}$ along the monopole track. Each nucleon decay is simulated as an electromagnetic cascade with an energy of $940 \mathrm{MeV}$, corresponding to the benchmark detection channel (Eq. 3). The simulation and propagation of the Cherenkov light from these cascades is done with the software package Photonics [48] using the ice model described in [49] for the IC-59 analysis and an improved version described in [50] for the IC-86/DeepCore analysis.

Background noise in the DOMs has to be superimposed on the signal. This noise consists of uncorrelated random noise, mostly from radioactive decays in the DOMs and correlated noise because of after pulses and signals from atmospheric muons. For the IC-59 analysis, the random noise is simulated as a Poisson process and the atmospheric muons are simulated using the software package CORSIKA [51] based on a 5-component model for cosmic rays with the hadronic interaction model SIBYLL [52] and the Hörandel flux model [53]. For the simulation of noise in the IC-86/DeepCore analysis the detector response of simulated monopole signals is superimposed with random and correlated noise hits from experimental data. These noise hits were recorded with a fixed rate trigger (FRT) that was implemented to measure and analyze background noise in the detector. More details on the FRT data are given in Sect. 3.2.

Figure 3 shows a simulated monopole event with $\beta=10^{-3}$ and $\lambda_{\text {cat }}=1 \mathrm{~cm}$. Because of the low speed, the event duration for a monopole is typically a factor of 1000 longer than for muon events and a large number of noise hits are recorded. However, the monopole also produces a large amount of Cherenkov light in the detector. Therefore, its signature can be separated from the randomly distributed noise hits already by eye. 


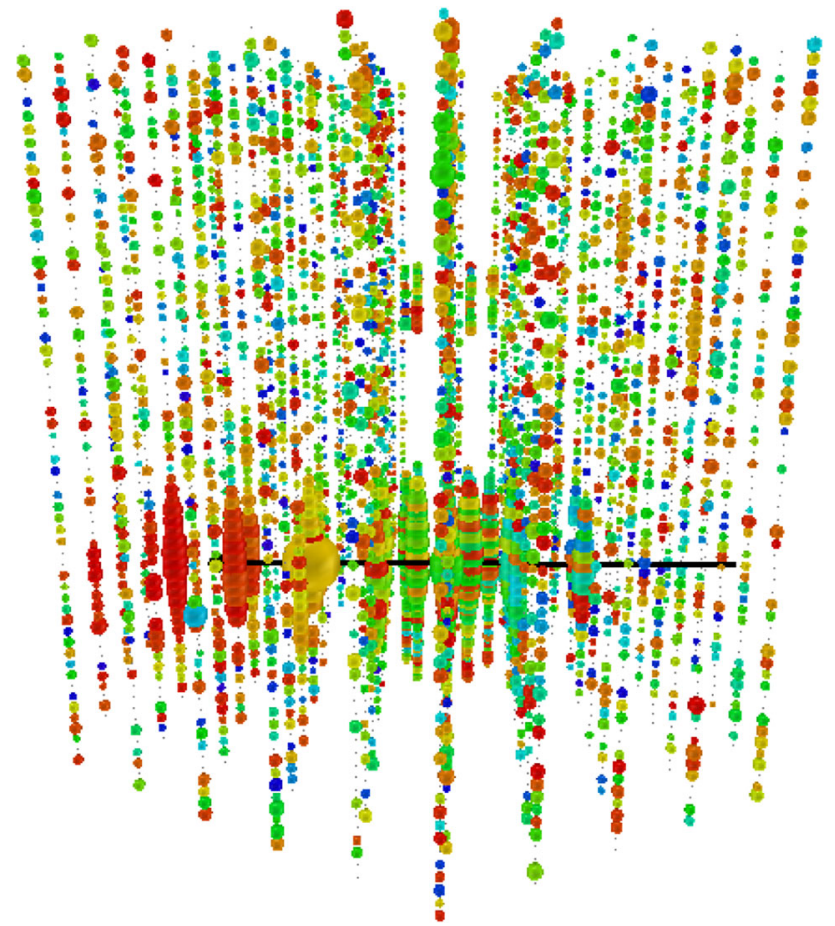

Fig. 3 Event display of a simulated monopole with $\beta=10^{-3}$ and $\lambda_{\text {cat }}=1 \mathrm{~cm}$ with superimposed background noise. The black line represents the monopole track. The DOMs are shown as tiny black dots. The color code illustrates the time scale from red for early times to blue for later times. The radii of the colored spheres scale with the number of recorded photo-electrons

\section{Search for magnetic monopoles with the slow particle trigger}

The experimental data set was recorded between May 2011 and May 2012 with a dedicated slow particle trigger applied to DeepCore. In this period the live time of the detector was 351 days, with a total number of approximately 50 million triggered events.

\subsection{The Slow-Particle Trigger}

Multiple IceCube triggers are implemented in the software of the data acquisition system [22]. Most of them are sensitive to signatures of relativistic particles, e.g. muons, so they have little sensitivity to non-relativistic magnetic monopoles. Only for the case of very bright magnetic monopoles the large amount of light can frequently prompt triggers for relativistic particles. This case is described in Sect. 4.1.

The Slow-Particle Trigger (SLOP trigger) was first implemented in May 2011 [54]. For the first year, the trigger operated only on the subdetector DeepCore. Since May 2012, the trigger has been operating on the full IceCube detector.

The SLOP-Trigger searches for time isolated local coincidences in nearby DOMs caused by subsequent nucleon

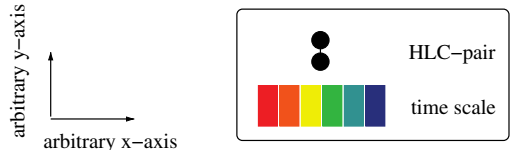

(a)

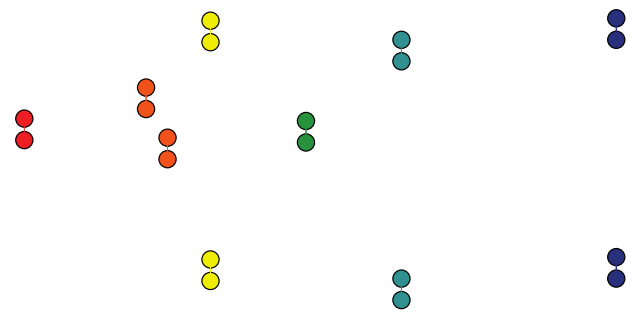

(b)

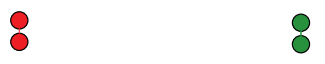

(c)

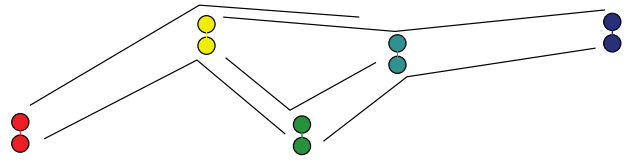

(d)

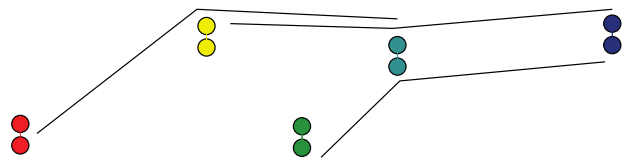

Fig. 4 Illustration of the SLOP trigger. The times and positions are arbitrary. The $x$ - and $y$-axis correspond to spatial coordinates and the color bar corresponds to a time scale. a List of all HLC pairs. For the trigger algorithm only the position and time of the first hit of each HLC pair is used. b The two HLC pairs (orange) with a time difference $\Delta t<t_{\text {proximity }}$ are removed. c All combinations of three HLC pairs, called triplet, with a time difference $\Delta t_{\mathrm{ij}} \in\left[t_{\min }, t_{\max }\right]$ between two pairs are built. $\mathbf{d}$ The cuts on the quality criteria $\Delta d$ and $v_{\text {rel }}$ remove two more triplets. If the remaining triplets overlap in time and fulfill $n$-triplet $\geq n_{\text {min }}$-triplet, a trigger is generated and the full detector data within the time span from the first to the last HLC pair of the triplets is recorded [54]

decays along the monopole trajetory. These coincidences have to be consistent with a straight particle track of constant speed.

The SLOP-Trigger is illustrated in Fig. 4. Specific values for the different trigger parameters are listed in Table 1. It is based on local coincidences of hits (HLCs, Sect. 2.1). For the trigger, the position and time, defined by the first hit of the HLC pair, of all HLC pairs are stored in a list (Fig. 4a). Since muons pass the detector within $\sim 5 \mu$ s they produce several HLC pairs within a short time. By removing all HLC pairs with time differences $\Delta t<t_{\text {proximity }}$ from the list, muon hits are efficiently rejected (Fig. $4 b$ ).

The remaining HLC pairs are searched for every combination of three HLC pairs, the triplets (Fig. 4c). The time difference between any two HLC pairs within a triplet has to be in the range $\left[t_{\min }, t_{\max }\right]$. Furthermore only triplets that 
Table 1 Trigger conditions of the SLOP-trigger [54]

\begin{tabular}{ll}
\hline Parameter & Value \\
\hline$t_{\text {proximity }}$ & $2.5 \mu \mathrm{s}$ \\
$t_{\min }$ & $0 \mu \mathrm{s}$ \\
$t_{\max }$ & $500 \mu \mathrm{s}$ \\
$\Delta d$ & $\leq 100 \mathrm{~m}$ \\
$v_{\text {rel }}$ & $\leq 0.5$ \\
$n_{\min }$-triplet & 3 \\
$L_{\max }$ & $5 \mathrm{~ms}$ \\
\hline
\end{tabular}

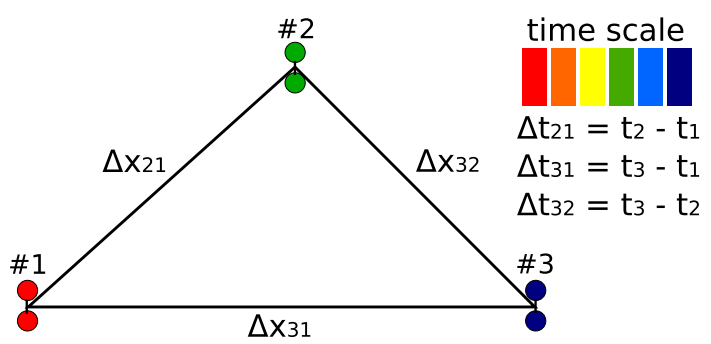

Fig. 5 Illustration of a triplet. All three HLC pairs are defined by the position $\left(\mathbf{x}_{1}, \mathbf{x}_{2}, \mathbf{x}_{3}\right)$ and the time $\left(t_{1}, t_{2}, t_{3}\right)$ of the first hit of an HLC pair. The trigger observables are the distances $\left(\Delta x_{21}, \Delta x_{32}, \Delta x_{31}\right)$ and time differences $\left(\Delta t_{21}, \Delta t_{32}, \Delta t_{31}\right)$

match a track-like signature are kept. Therefore two quality criteria are required: the contributing HLC pairs have to be ordered along a line and the time differences have to be consistent with a constant speed (Fig. 5).

The first can be verified by the parameter $\Delta d=\Delta x_{21}+$ $\Delta x_{32}-\Delta x_{31}$. If $\Delta d=0$ all HLC pairs are located on a line. The second can be checked by the parameter

$v_{\text {rel }}=\frac{\left|\frac{1}{v_{21}}-\frac{1}{v_{32}}\right|}{\frac{1}{3} \cdot\left(\frac{1}{v_{21}}+\frac{1}{v_{32}}+\frac{1}{v_{31}}\right)}$,

where $v_{i j}=\frac{\Delta x_{i j}}{\Delta t_{i j}}$ with $i, j \in\{1,2,3\}$ corresponds to the speed between the $j$ th and the $i$ th HLC pair within a triplet. For a monopole with a constant speed all HLC pairs should be connected by a constant speed and therefore $v_{\text {rel }} \rightarrow 0$ should be valid. All triplets not satisfying these quality criteria are removed from the set of triplets (Fig. 4d).

Finally, if the number of triplets in the set overlapping in time, $n$-triplet, is greater than a minimum number of triplets $n_{\text {min }}$-triplet, the trigger is launched. When these conditions are met, the full detector data from the first to the last HLC pair in the list of triplets are stored, also including those DOM signals not contributing to the trigger. The maximum event duration of the trigger is restricted to $L_{\max }=5 \mathrm{~ms}$.

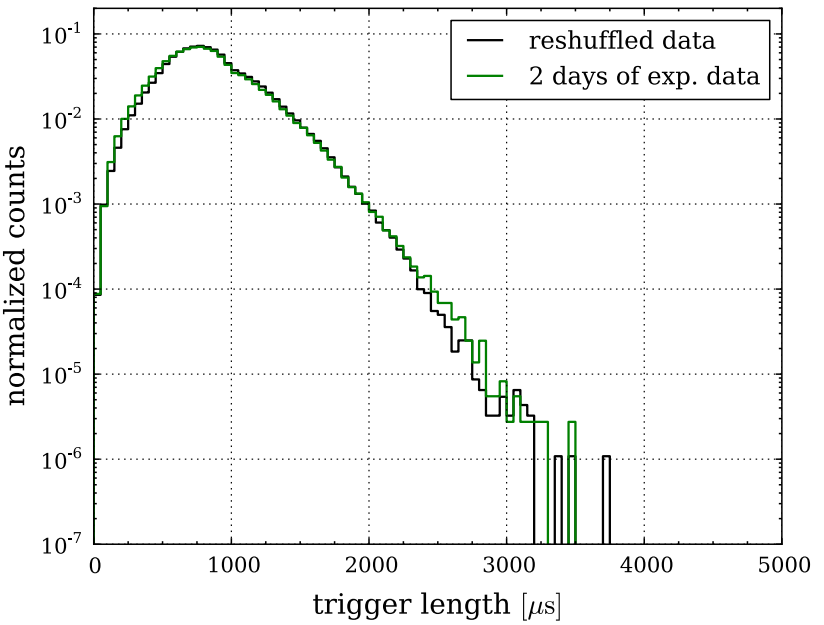

Fig. 6 Event duration distribution of an experimental 2 days data set (green). The trigger rate is $2.1 \mathrm{~Hz}$. The maximum is at about $750 \mu \mathrm{s}$. For comparison the event duration distributions of the generated background events (black) is superimposed. The number of entries is normalized to one

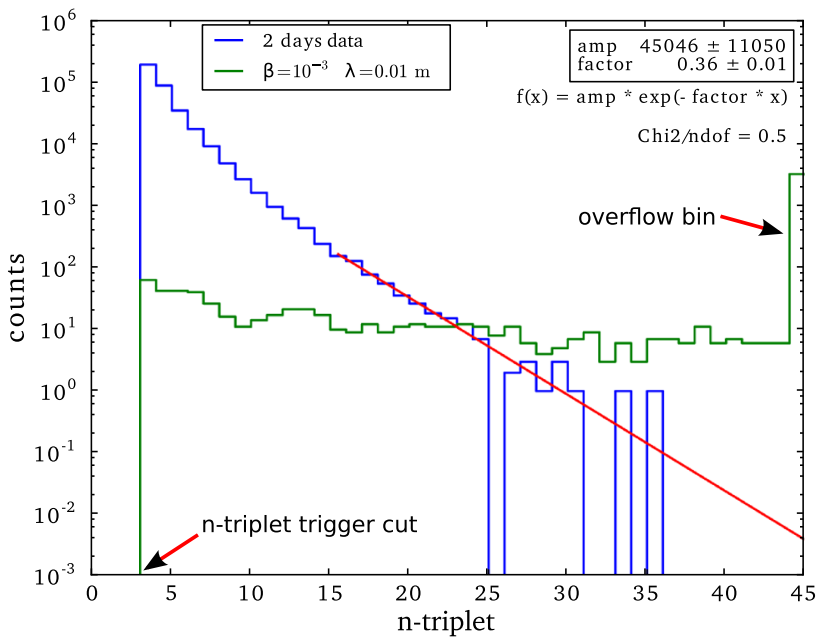

Fig. $7 n$-triplet distribution of the experimental test data sample (blue) in comparison to a distribution of simulated monopoles with $\beta=10^{-3}$ and $\lambda_{\text {cat }}=1 \mathrm{~cm}$ (green). In addition, an exponential function is fitted to the tail of the experimental distribution for $n$-triplet $\geq 15$ (red)

\subsection{Background study for the SLOP data}

To investigate the characteristics of the SLOP events, we use an experimental data set of $\sim 2$ days of live time. This is sufficiently short to exclude a significant signal contamination given by current flux limits (Sect. 1) and hence the data can be considered as background.

Figure 6 shows the distribution of event durations of SLOP triggered events. Typical durations are of the order of milliseconds, whereas the other IceCube triggers have typical durations of a few microseconds.

Figure 7 compares the $n$-triplet distribution of the experimental data sample with simulated monopoles of 


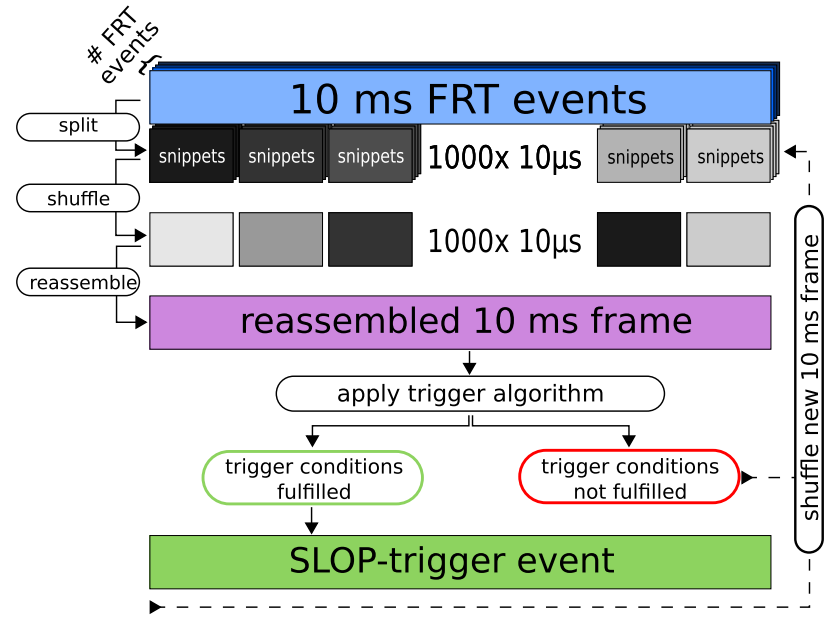

Fig. 8 Illustration of the generation of background events by reshuffling experimental data measured by a fixed rate trigger (FRT). FRT events have a length of $10 \mathrm{~ms}$. They are split into $10 \mu \mathrm{s}$ snippets. The snippets are shuffled randomly to build new $10 \mathrm{~ms}$ frames. Then the SLOP trigger algorithm is applied

$\beta=10^{-3}$ and $\lambda_{\text {cat }}=1 \mathrm{~cm}$. While the background distribution decreases rapidly for larger $n$-triplet, the signal distribution is almost flat. Therefore, the quantity $n$-triplet discriminates well between signal and background events. The exponential decrease of the background distribution indicates a possible Poissonian random process for combinations of HLC pairs which result in a triplet.

To understand the underlying random processes for the background events we developed a method to generate a high statistics sample of background events by reshuffling experimental events recorded with the FRT. The FRT fires at fixed time intervals (e.g., every $30 \mathrm{~s}$ ), and DOM data from the entire detector are recorded over a time interval of $10 \mathrm{~ms}$. The resulting events contain all types of random and correlated backgrounds, and highly unlikely any signal.

The FRT events of $10 \mathrm{~ms}$ length were split into snippets of $10 \mu \mathrm{s}$, which were then randomly re-ordered to form new $10 \mathrm{~ms}$ events. The newly assembled events are then passed to the SLOP trigger algorithm (Fig. 8). This way, a total of $400 \mathrm{~s}$ of FRT data were re-shuffled to generate a background sample of about 25 days of live-time equivalent. The generated sample closely resembles the experimental SLOPtriggered events. Figure 6 compares the event duration of the generated background events to the SLOP-triggered events in 2 days of experimental data. The method reproduces the measured event duration distribution reasonably well over several orders of magnitude. For shorter event duration the distribution of the generated data sample tends to be below the distribution of the experimental test data sample. This is expected because this method cannot correctly model noise hits that are correlated over time scales larger than the length of the $10 \mu \mathrm{s}$ snippets. Below $10 \mu \mathrm{s}$ the triplets are characterized by the same DOM combinations due to the low

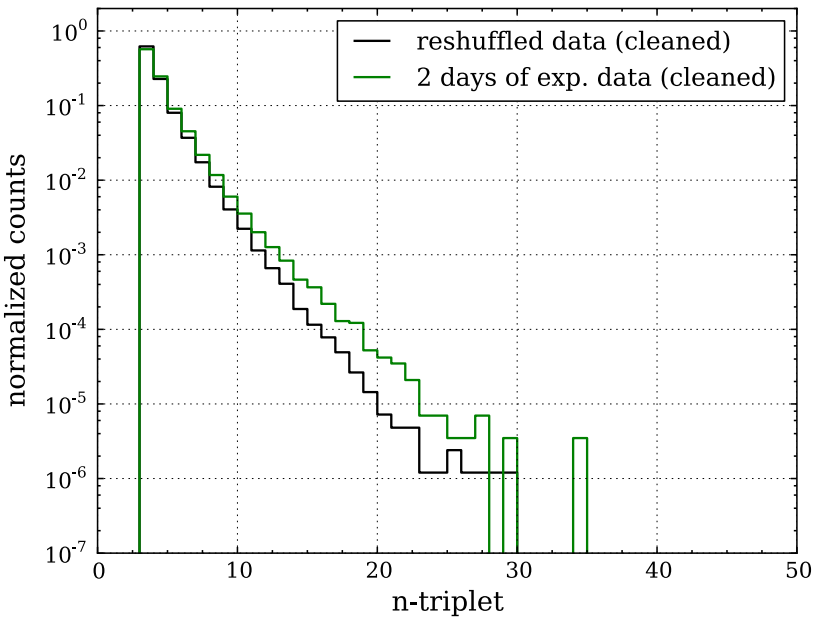

Fig. 9 Comparison of the $n$-triplet distributions of the experimental test data set (green) and the generated background events (black). Triplets caused by HLC pairs fulfilling $\Delta t_{21}$ or $\Delta t_{32} \leq 50 \mu \mathrm{s}$ are not taken into account (cleaned)

statistics of the FRT events. The overall good agreement indicates that correlated noise is a subdominant effect and is only relevant for short time scales. We will presume later that different triplets due to correlated noise are themselves based on largely independent sets of HLC pulses. Therefore, for large values of $n$-triplet the contribution from correlated noise triplets is added as a random process similar to the triplets from uncorrelated noise.

Figure 9 compares the $n$-triplet distributions of experimental data and generated background. Overall both distributions are similar and show an exponential decay. The differences can be understood by two effects. The first is the aforementioned effect that noise correlations over time scales longer than $10 \mu \mathrm{s}$ are not taken into account, which is expected to increase the number of triplets. By removing triplets which arise from HLC pairs fulfilling the typical time scale of the correlated noise $\left(\Delta t_{21}\right.$ or $\left.\Delta t_{32} \leq 50 \mu \mathrm{s}\right)$ the agreement improves. However, overall correlated noise has only a small effect on these distributions. More importantly, the FRT data and the SLOP test data do not correspond to the same data taking period. The DOM noise rate shows slow slight drifts over long periods of time. The chance probability of producing large $\mathrm{n}$-triplet values depends on this random noise. This effect is accounted for by the background fit described in the following section. In conclusion, the observed background is understood by the noise characteristics of the DOMs.

\subsection{Background model for the SLOP data}

As a result of the findings in the previous section, the $n$ triplet distribution for the background is estimated by fitting the experimental data with a simple probabilistic model. 
The generic ansatz assumes that the probability to find a triplet (3 HLC pairs) can be described with a combinatorial model. For a number of $N$ HLC pairs the maximum number of possible triplets is given by $n_{\max }=\left(\begin{array}{c}N \\ 3\end{array}\right)$. If the probability $p$ for any three out of $N$ HLC pairs to build a triplet is approximately constant, then the probability for $n$ triplets out of $n_{\max }$ possible triplets follows the binomial distribution:

$B\left(n \mid n_{\max }, p\right)=\left(\begin{array}{c}n_{\max } \\ n\end{array}\right) p^{n}(1-p)^{n_{\max }-n}$.

As the HLC pairs themselves arise from random noise, the probability to observe $N$ HLC pairs is given by a Poissonian:

$P_{\mu}(N)=\frac{\mu^{N}}{N !} \mathrm{e}^{-\mu}$,

where $\mu$ is the mean expectation for the number of HLC pairs $N$ in a given time window. The total probability to observe the number $n$ triplets is given by a sum over all binomial probabilities $B\left(n \mid n_{\max }(N), p\right)$ weighted with the probability to observe $N$ HLC pairs. This results in

$$
P(n \mid \mu, p)=P_{0} \sum_{N=N_{\min }(n)}^{\infty} P_{\mu}(N) \cdot B\left(n \mid n_{\max }(N), p\right) .
$$

The sum starts with the minimum number of HLC pairs $N_{\text {min }}(n)$ which are combinatorially required for $n$ triplets. This is given by the solution of the equation $n=\left(\begin{array}{c}\left\lfloor N_{\min }\right\rfloor \\ 3\end{array}\right)$. Here, $\left\lfloor N_{\min }\right\rfloor$ is the greatest integer less than or equal to $N_{\min }$. The parameter $P_{0}$ is the total normalization of $P(n \mid \mu, p)$.

With this ansatz it is possible to describe the distribution of $n$-triplet with only three parameters $P_{0}, \mu$ and $p$. Figure 10 shows the fit of this model to two normalized, experimental

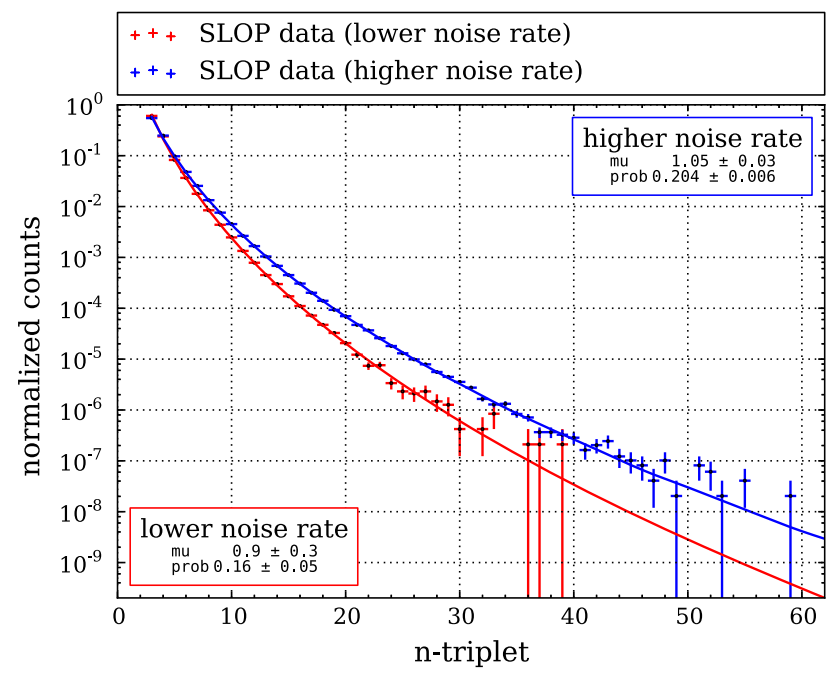

Fig. $10 n$-triplet distributions of experimental SLOP data. The blue distribution corresponds to $\mathrm{a} \sim 15 \%$ higher noise rate than the red one. Both distributions are normalized to one. The solid lines show the results of the background model fit and the fit parameters $\mu$ and $p$ are shown in the boxes $n$-triplet distributions which are based on SLOP data corresponding to different noise rates. Since the distributions are normalized only $\mu$ and $p$ have to be fit. The background model well describes the $n$ triplet distributions over several orders of magnitude. Moreover the increase in the noise rate is reflected in an increase of the value of $\mu$, which depends on the noise rate. In summary it can be confirmed that the background events from the SLOP trigger are dominated by random noise.

\subsection{Reconstruction of a monopole track}

The analysis searches for monopoles from all directions. Also the random background is largely isotropic and the event selection does not depend specifically on the direction of the monopole. However, an important observable is the speed of the track, which can be estimated with the line fit [55]. This algorithm is based on a simple ansatz in which the geometry of the Cherenkov cone and the optical properties of the medium are ignored and the particle is assumed to travel with a velocity $\mathbf{v}$ along a straight line through the detector. A pseudo- $\chi^{2}$ is constructed with the positions $\mathbf{x}_{i}$ and times $t_{i}$ of the HLC pairs of all $N$ selected triplets:

$\chi^{2}=\sum_{i}^{N} \frac{\left|\mathbf{x}_{i}-\left(\mathbf{x}_{0}+\mathbf{v} \cdot t_{i}\right)\right|^{2}}{1 \mathrm{~m}^{2}}$.

HLC pairs which participate in multiple triplets are taken into account multiple times. This $\chi^{2}$ can be minimized analytically with respect to the speed $\mathbf{v}$ and vertex $\mathbf{x}_{0}$. Note that $\chi^{2}$ is arbitrarily normalized and cannot be interpreted statistically in terms of goodness of fit. The following analysis uses only the estimated speed $|\mathbf{v}|$.

In Fig. 11 the distributions of the reconstructed speeds are shown. The reconstructed speeds are a reasonable estimate of the true speed, in particular for faint monopoles (see $\lambda_{\text {cat }}=1 \mathrm{~m}$ ). For brighter monopoles (see $\lambda_{\text {cat }}=1 \mathrm{~cm}$ ), the reconstructed speeds slightly underestimate the true speed.

This reconstruction algorithm is simple, robust, and fast, while still yielding a sufficient accuracy. It also allows us to approximate the monopole direction by the direction of $\mathbf{v}$. The mean difference between the true and reconstructed direction varies between $\sim 11^{\circ}$ and $\sim 20^{\circ}$ depending on the monopole speed and the mean free path $\lambda_{\text {cat }}$.

\subsection{Event selection and background reduction}

For this first IceCube analysis of SLOP data a robust approach based on $n$-triplet as the single final selection criterion and the determination of the expected background from experimental data was chosen.

Figure 12 shows the probability density distributions of $n$-triplet for events with a reconstructed speed of at least 

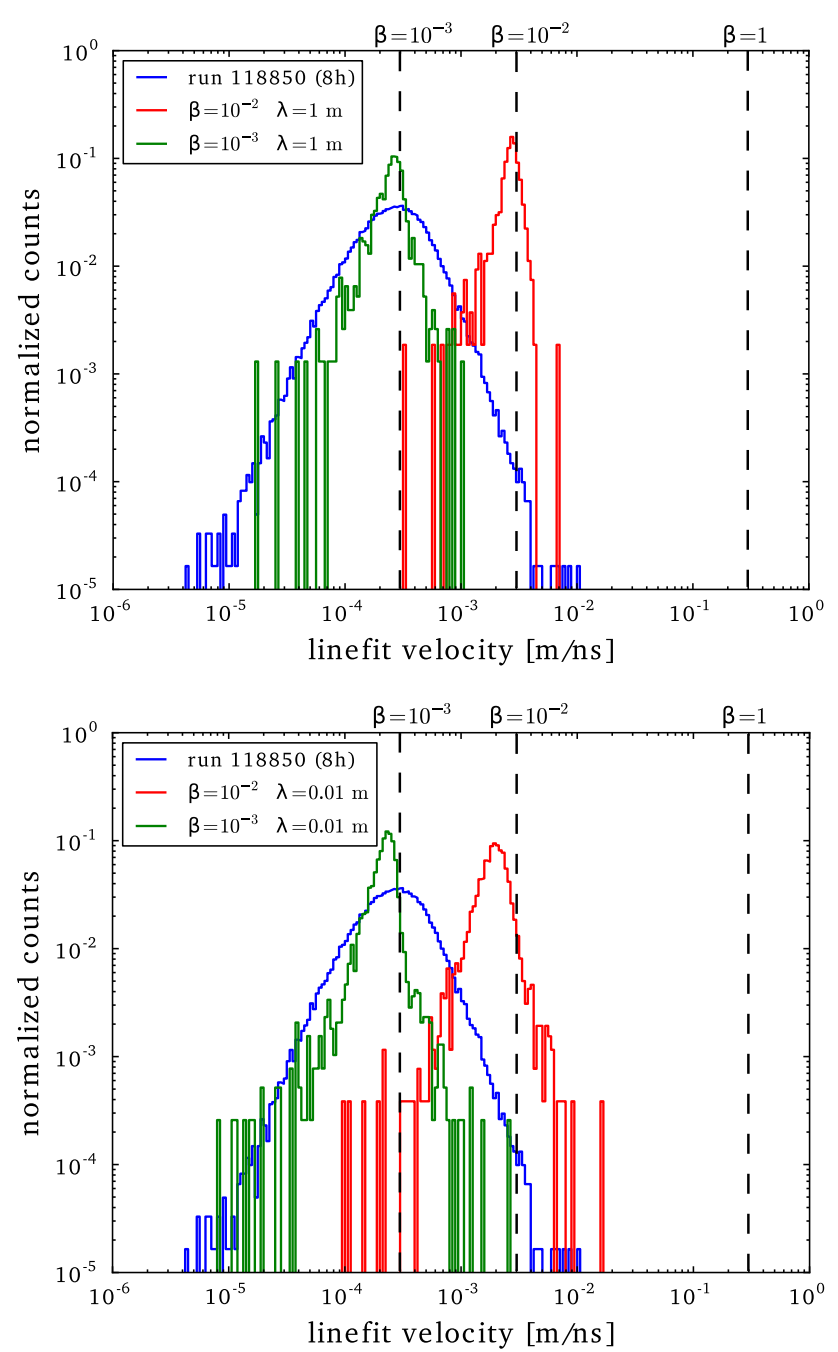

Fig. 11 Distribution of the reconstructed speeds for two different simulated monopole speeds. At the top the distributions for monopoles with $\lambda_{\text {cat }}=1 \mathrm{~m}$ and at the bottom for monopoles with $\lambda_{\text {cat }}=1 \mathrm{~cm}$ are shown. For comparison, reconstructed experimental data corresponding to a live time of $8 \mathrm{~h}$ are plotted. The three dotted black lines show the true speeds and the speed of light. All distributions are normalized to one

$10^{-3} \mathrm{~m} / \mathrm{ns}$ (top) and with a reconstructed speed less than $10^{-3} \mathrm{~m} / \mathrm{ns}$ (bottom). While the signal expectation extends to very high $n$-triplet, the distributions of the experimental data decrease rapidly. The final cuts on $n$-triplet were optimized for maximum sensitivity based on the Model Rejection Factor [56]. The optimization resulted in the following criteria/cuts: $n$-triplet $\geq 60$ for a reconstructed speed $v<10^{-3} \mathrm{~m} / \mathrm{ns}$ and $n$-triplet $\geq 26$ for $v \geq 10^{-3} \mathrm{~m} / \mathrm{ns}$.

These selection cuts were defined before unblinding the full experimental data. Here, an iterative two step procedure was chosen. First, $10 \%$ experimental data was unblinded with the selection determined by the aforementioned experimental 2 days data sample. After no signal or unexpected background was observed the same procedure was applied to the full experimental data.
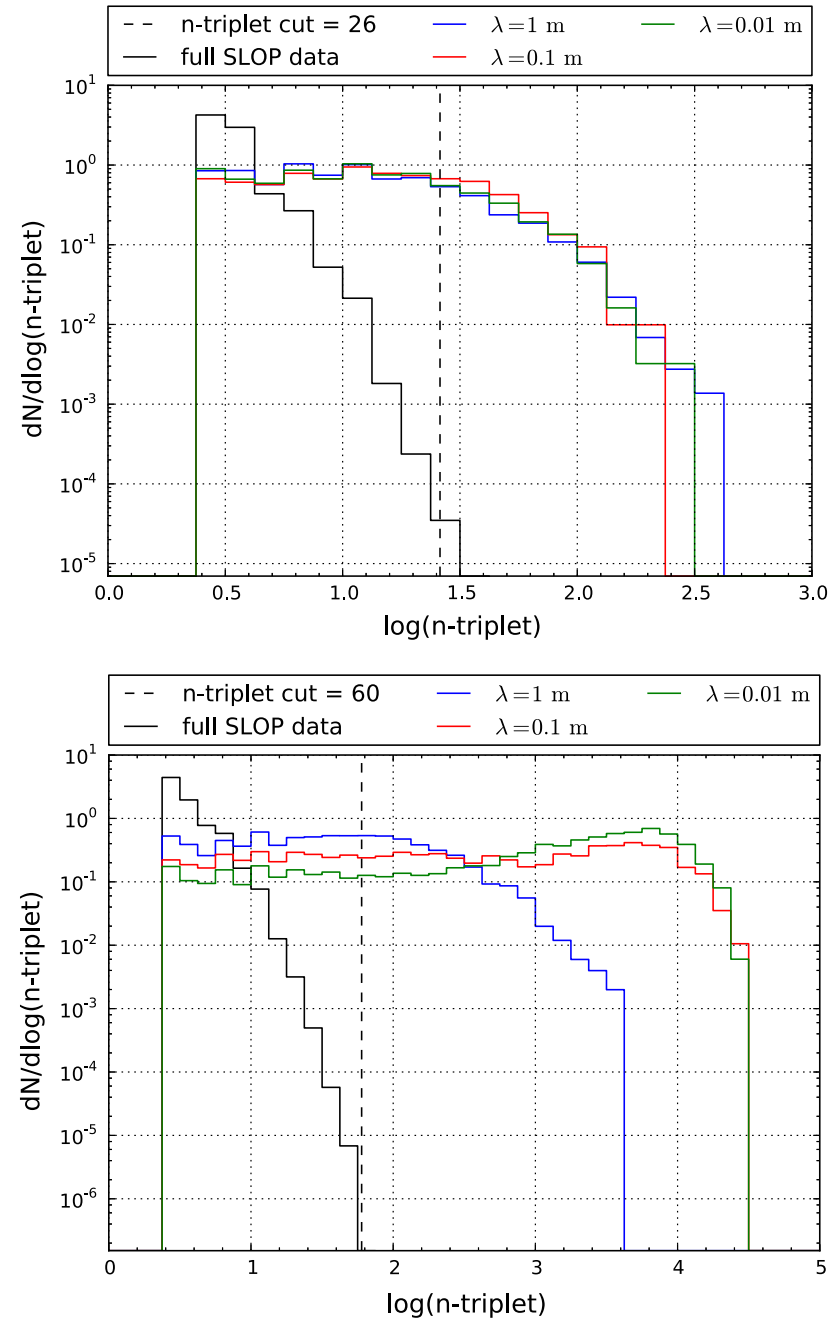

Fig. 12 Probability density distributions of $n$-triplet for events with larger reconstructed speed (top) and for events with smaller reconstructed speed (bottom). In black the distributions of 1 year experimental data are shown. The signal distributions are shown with decreasing $\lambda_{\text {cat }}$ in blue, red, and green. The final cuts on $n$-triplet are shown by the dashed black line

Figure 13 shows the resulting $n$-triplet-speed distribution for the full year of experimental data. After the final selection only one experimental event with $n$-triplet $=34$ and $v=1.15 \times 10^{-3} \mathrm{~m} / \mathrm{ns}$ remains, but not well separated from the background. Closer inspection revealed no evidence for an obvious track-like signature, in particular most triplets would not have survived tighter causality requirements. As this observation is consistent with the expected number of about three background events (see below), we do not consider this result as positive detection.

\subsection{Results}

With no observed monopole signal we have derived an upper limit on the flux of non-relativistic magnetic monopoles 


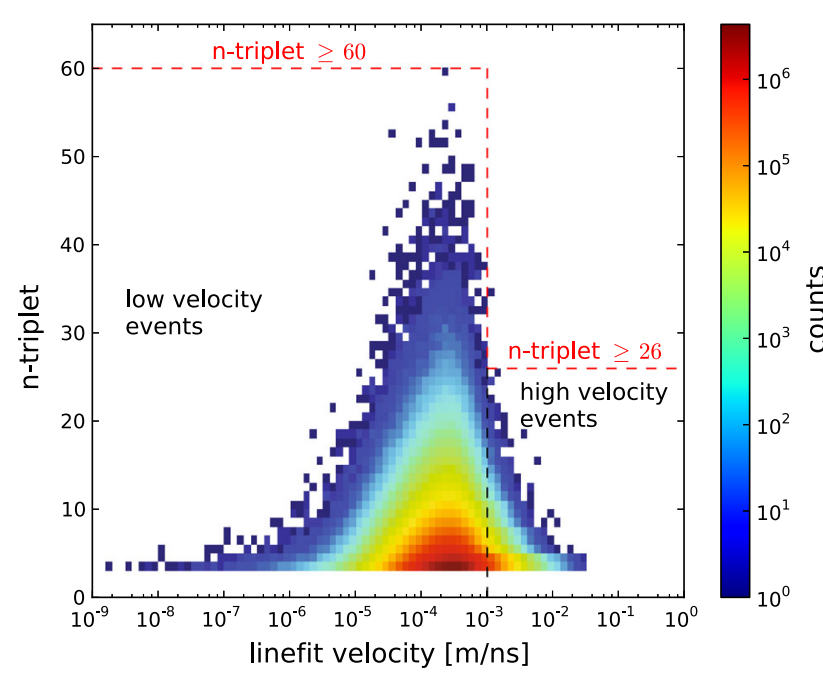

Fig. $13 n$-triplet-speed distribution of 1 year of experimental data. The final cuts on $n$-triplet are shown by the dashed red lines. The boundary between the two speed regions is shown by the dashed black line

(Sect. 6). For this, the background model is fit to the $n$-triplet distributions for both speed ranges (Fig. 14). It is found that the background model (Sect. 3.3) well describes the $n$-triplet distribution over several orders of magnitude.

Based on the fit results, the expected number of background events with $n$-triplet $\geq n_{\text {cut }}$ can be calculated by the integral

$n_{\mathrm{b}}^{\text {fast } / \text { slow }}=\int_{n_{\text {cut }}}^{\infty} P\left(n \mid \mu^{\text {fast/slow }}, p^{\text {fast } / \text { slow }}\right) d n$.

The total expected number of background events is defined by the sum of the expectation of both speed regions $n_{\mathrm{b}}=$ $n_{\mathrm{b}}^{\text {fast }}+n_{\mathrm{b}}^{\text {slow }}$. By varying the fit parameters within their fitted uncertainty a pseudo-experiment with different expected numbers of background events can be performed. Figure 15 shows the resulting probability density distribution of the total expected number of background events. The median expected number of background events is $n_{\mathrm{b}}^{\text {median }}=3.2_{-1.1}^{+1.8}$. Here, the statistical uncertainty is approximated by the difference between the median and the quantiles $Q_{0.16}$ and $Q_{0.84}$.

\section{Search for very bright magnetic monopoles with the IC-59 array}

The search for magnetic monopoles presented in this section uses data taken during the season 2009-2010 when IceCube was running in its 59-string configuration. This analysis used the data taken with the standard IceCube triggers. The standard trigger that is used for highly energetic relativistic particles is a simple multiplicity trigger (SMT), which requires at least eight HLC hits within a sliding time window of $5 \mu s$
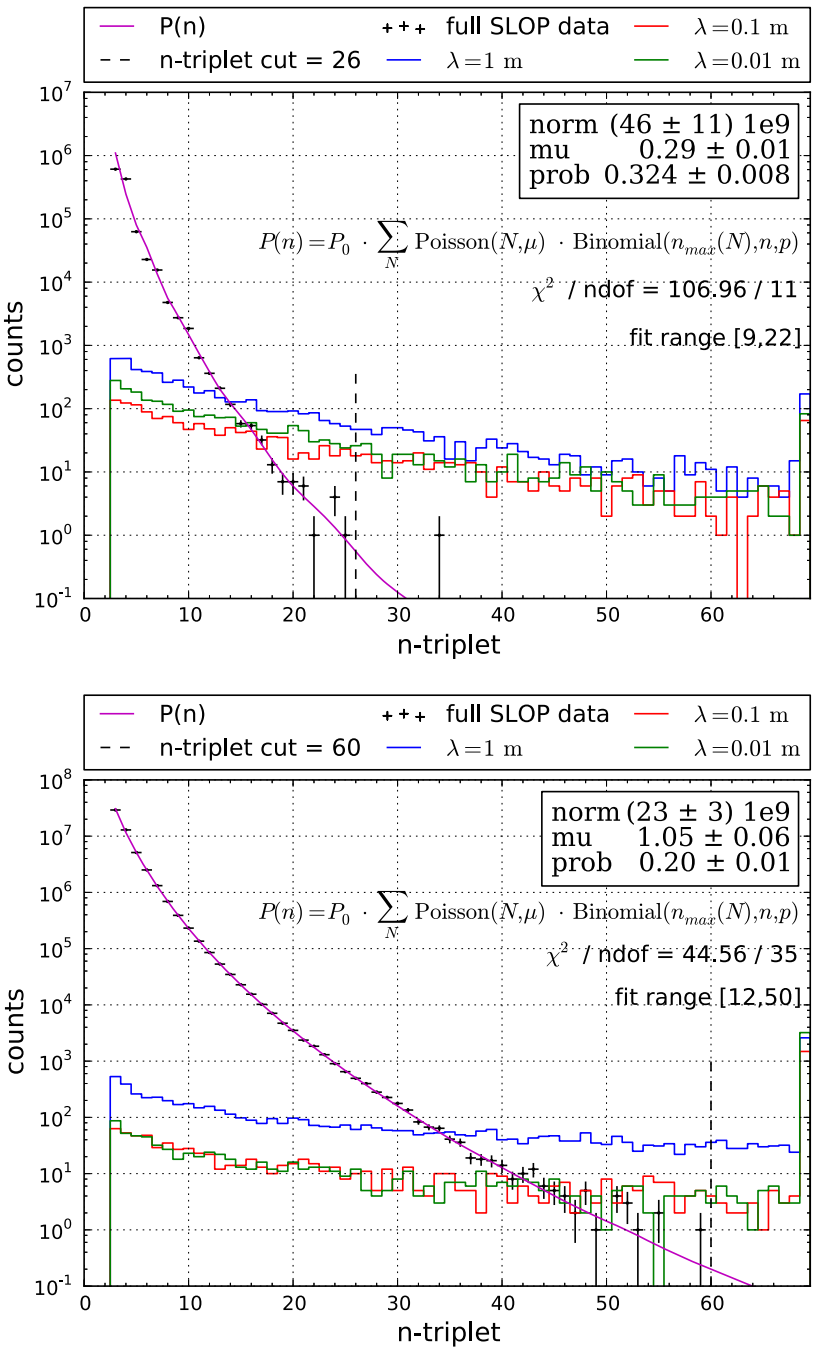

Fig. $14 n$-triplet distributions for events with larger reconstructed speed (top) and for events with a smaller reconstructed speed (bottom). The black data points show the distributions of the full experimental data. The expected signal is shown with decreasing $\lambda_{\text {cat }}$ in blue, red and green. The fitted functions $P(n \mid \mu, p)$ are shown in purple and the final selections on $n$-triplet as dashed black lines

(SMT-8). Other triggers are optimized for relativistic particles with lower energies. Data are recorded over at least the time interval over which the trigger condition of any of the triggers is fulfilled. For HLC hits, the full PMT waveforms are digitized and recorded [22]. Not all triggered events were transmitted to the Northern hemisphere by satellite. Events of various categories (e.g. track-like, cascade-like, very bright events, etc.) have been selected by various online filters at the South Pole [57,58]. Although the filters are optimized for relativistic particles, they may accept bright monopole events if a sufficient number of DOMs are hit. This analysis uses the cascade and high-energy filters, which have the best acceptance for non-relativistic monopoles. The total live time of this data set is 311.25 days, with an average rate of 


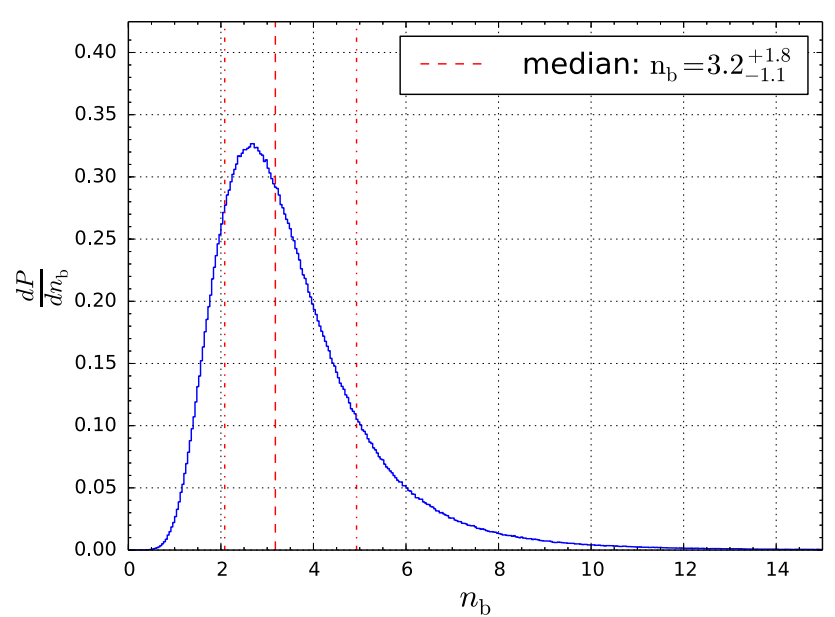

Fig. 15 Probability density distribution of the expected number of background events. The median is shown by the dashed red line. The quantiles $Q_{0.16}$ and $Q_{0.84}$ are shown by the dash-dotted red line

selected events of $85.5 \mathrm{~Hz}$. The efficiency of this filter selection with respect to the multiplicity trigger is above $75 \%$ for monopoles of $\beta=10^{-3}$ and $\lambda_{\text {cat }}=1 \mathrm{~mm}$.

\subsection{Selection of very bright magnetic monopoles}

Slow monopoles with a catalysis cross section $\sigma_{\text {cat }}$ much larger than $10^{-23} \mathrm{~cm}^{2}$ appear as very bright tracks. Simulations of the detector response to such tracks show that the multiplicity condition is fulfilled over most of the monopole crossing time, or that successive triggers occur close enough in time for the recording intervals to overlap. So, a large fraction of a monopole's catalysis signature would be captured in a single event, if $\sigma_{\text {cat }}$ is sufficiently high. For $\sigma_{\text {cat }}<10^{-23}$ $\mathrm{cm}^{2}$ monopoles still yield multiple triggers, but the triggers occur less frequently, so that the signature is often split up into several subevents. The smaller the cross section, the more the monopole event splits up and the larger are the gaps between the subevents. Eventually, the signal becomes indistinguishable from the background. Therefore, this analysis focuses on catalysis cross sections above $10^{-23} \mathrm{~cm}^{2}$. For monopoles with such high $\sigma_{\text {cat }}$, the IC-59 analysis achieves a better sensitivity than the analysis using the SLOP trigger. This is simply because the IC-59 array had a much larger detection volume than the DeepCore array available to the previously described analysis. Future monopole searches will use data taken after 2012, when the SLOP trigger was operating on the full IC-86 array. These analyses will take advantage of both the large detection volume of the full IC-86 array and the high efficiency of the SLOP trigger.

\subsection{IC-59 background reduction}

The high-energy and cascade filters provide a data sample with about $10^{9}$ events. The vast majority of these events are down-going atmospheric muons. This background is reduced using a set of straight cuts in a first step. These cuts are based on the time and location of the detected Cherenkov photons. Contrary to the IC-86/DeepCore analysis, whose cut parameters where defined using the time and location of DOM launches or HLC pairs, this analysis uses a feature extraction algorithm on the PMT waveforms, which reconstructs the constituent PMT pulses caused by individual photo electrons. In a second step a Multivariate Analysis is adopted to reduce the background further.

The variables used for background reduction are:

1. The event duration $\Delta t$ defined as the time difference between the last and first pulse registered by a DOM in an event.

2. The reconstructed speed $\mathrm{v}$ from the line fit.

3. The number of clusters ( $\left.N_{\text {clusters }}\right)$, which is defined by the reconstructed pulses on all DOMs sorted into groups of pulses which occur close in space and time. Each such group is called a cluster and the total number of these clusters in an event is used as a cut variable. Bright signal tracks tend to have a higher number of clusters than atmospheric muon background events.

4. The total number of photo-electrons collected in the whole detector divided by the event duration, $Q_{\mathrm{tot}} / \Delta \mathrm{t}$.

5. Median of the distance between clusters along the reconstructed track.

6. The center of gravity (COG) of the event, defined as the average spatial coordinates of all hits.

Straight cuts are applied to variables $1-5$, chosen to substantially reduce the background while keeping the signal efficiency reasonably high (Table 2). The cut on variable 6 removes events that only traverse a corner of the detector.

After applying those cuts, a Multivariate Analysis is performed on the remaining data to define a final selection criterion. In addition to variables 3, 4, and 5 this Multivariate Analysis considers the following variables:

7. Mean distance of the hit DOMs to the center of gravity (COG) of the event divided by the event duration.

8. Number of clusters divided by the event duration.

9. Number of simple multiplicity triggers divided by the number of strings with hit DOMs

\subsection{Signal expectations}

Data are divided into two sets according to the monopole track brightness (i.e. the catalysis cross section). The cross section values for which we optimized the analysis and derive flux limits are $\sigma_{\text {cat }}=1.7 \cdot 10^{-22} \mathrm{~cm}^{2}$ and $\sigma_{\text {cat }}=$ 
Table 2 Signal efficiencies, data reduction factors and data rates before and after each cut for both $\sigma_{\text {cat }}\left(\lambda_{\text {cat }}\right)$. For $\sigma_{\text {cat }}=1.7 \cdot 10^{-22} \mathrm{~cm}^{2}$ the corresponding applied cuts are "Cut 1 to Cut 6 " which are described in subsection 4.2. For $\sigma_{\text {cat }}=1.7 \cdot 10^{-23} \mathrm{~cm}^{2}$ the applied cuts are: Cut 1 , Cut 2 and Cut 6

\begin{tabular}{|c|c|c|c|c|c|c|c|}
\hline & Before the cut & Cut 1 & Cut 2 & Cut 3 & Cut 4 & Cut 5 & Cut 6 \\
\hline \multicolumn{8}{|c|}{$\sigma_{\text {cat }}=1.7 \cdot 10^{-22} \mathrm{~cm}^{2}, \lambda_{\text {cat }}=1 \mathrm{~mm}$} \\
\hline$\beta=10^{-2}$ & $57 \%$ & $43.5 \%$ & $42.3 \%$ & $41.9 \%$ & $41.9 \%$ & $41.8 \%$ & $33.6 \%$ \\
\hline$\beta=10^{-3}$ & $75.4 \%$ & $45.3 \%$ & $41.1 \%$ & $41 \%$ & $41 \%$ & $39.8 \%$ & $34.3 \%$ \\
\hline Experiment: reduction factor & - & $8 \cdot 10^{-4}$ & $7.8 \cdot 10^{-5}$ & $6.6 \cdot 10^{-5}$ & $5.7 \cdot 10^{-5}$ & $5.3 \cdot 10^{-5}$ & $4.8 \cdot 10^{-5}$ \\
\hline Experiment: rate $\left(\mathrm{s}^{-1}\right)$ & 85.5 & $6.8 \cdot 10^{-2}$ & $6.7 \cdot 10^{-3}$ & $5.6 \cdot 10^{-3}$ & $4.9 \cdot 10^{-3}$ & $4.5 \cdot 10^{-3}$ & $4.1 \cdot 10^{-3}$ \\
\hline \multicolumn{8}{|c|}{$\sigma_{\text {cat }}=1.7 \cdot 10^{-23} \mathrm{~cm}^{2}, \lambda_{\text {cat }}=1 \mathrm{~cm}$} \\
\hline$\beta=10^{-2}$ & $41 \%$ & $17.2 \%$ & $17 \%$ & $13.9 \%$ & & & \\
\hline$\beta=10^{-3}$ & $43 \%$ & $3.5 \%$ & $3.23 \%$ & $3.1 \%$ & & & \\
\hline Experiment: reduction factor & - & $1.4 \cdot 10^{-3}$ & $3.9 \cdot 10^{-4}$ & $3.45 \cdot 10^{-4}$ & & & \\
\hline Experiment: rates $\left(\mathrm{s}^{-1}\right)$ & 85.5 & $1.2 \cdot 10^{-1}$ & $3.3 \cdot 10^{-2}$ & $2.95 \cdot 10^{-2}$ & & & \\
\hline
\end{tabular}

$1.7 \cdot 10^{-23} \mathrm{~cm}^{2}$, which correspond to $\lambda_{\text {cat }}=1 \mathrm{~mm}$ and $\lambda_{\text {cat }}=1 \mathrm{~cm}$, respectively.

Figure 16 compares event duration $\Delta \mathrm{t}$ and reconstructed speed $\mathrm{v}$ of experimental data to those of bright monopoles with simulated $\lambda_{\text {cat }}=1 \mathrm{~mm}$ and speeds $\beta$ of $10^{-2}$ and $10^{-3}$. The signal efficiencies are 57.0 and $75.4 \%$ at the filter level for $\beta=10^{-2}$ and $\beta=10^{-3}$, respectively. A cut $\Delta \mathrm{t}>30 \mu \mathrm{s}$ reduces the data by a factor $8 \cdot 10^{-4}$ while keeping $43.5 \%$ of the signal for $\beta=10^{-2}$ and $45.3 \%$ for $\beta=10^{-3}$ at the filter level. Note that the average duration of triggered events is shorter for slower speeds than for faster, because slower monopole events are more likely to be split into multiple subevents.

Relativistic single-muon tracks have a reconstructed speed $\mathrm{v}$ around $0.3 \mathrm{~m} / \mathrm{ns}$. Events having passed the preceding cut on the time duration $\Delta \mathrm{t}$ are enriched with coincident muons from uncorrelated air showers, resulting in a lower $\mathrm{v}$ (see Fig. 16, bottom). For monopoles, the speed $v$ is close to the simulated values. Cutting at $\mathrm{v}<9 \cdot 10^{-3} \mathrm{~m} / \mathrm{ns}$ (corresponding to $\beta<3 \cdot 10^{-2}$ ) reduces the background by another order of magnitude.

Further cuts on variables 3-6 reduce the background by another factor two. In total after this first set of cuts the data rate is reduced by a factor $5 \cdot 10^{-5}$ while the signal efficiencies only drop to 33.6 and $34.3 \%$ for $\beta=10^{-2}$ and $\beta=10^{-3}$, respectively. Data reduction factors, rates and signal efficiencies before and after each applied cut are presented in Table 2.

For a ten times lower $\sigma_{\text {cat }}$, the monopole tracks are dimmer and the signal efficiency drops dramatically. Before applying any cut, the efficiencies at the filter level are 41 and $43 \%$ for $\beta=10^{-2}$ and $\beta=10^{-3}$, respectively. Figure 17 compares the same variables presented in Fig. 16. Signal and background are much less separated than for $\lambda_{\text {cat }}=1 \mathrm{~mm}$. Moreover, none of the events with $\beta=10^{-3}$ has a dura- tion that exceeds $800 \mu \mathrm{s}$, which is much less than the $3 \mathrm{~ms}$ necessary to cross the full array; i.e. most events are split into one or more subevents which have a shorter event duration in comparison to $\lambda_{\text {cat }}=1 \mathrm{~mm}$. The properties of these subevents are determined by hits from muons and from noise falling in the time window of the monopole passage. Thus the cuts applied on $\Delta \mathrm{t}$ and $\mathrm{v}$ had to be slightly relaxed compared to $\lambda_{\text {cat }}=1 \mathrm{~mm}: \Delta \mathrm{t}>28 \mu s$ and $\mathrm{v}<1.5 \cdot 10^{-2} \mathrm{~m} / \mathrm{ns}$. After excluding events with a reconstructed center of gravity (COG) at outer strings, the data rate is reduced by a factor $3.45 \cdot 10^{-4}$. Table 2 shows the data reduction factors, rates, and the final signal efficiencies before and after each applied cut. The signal efficiencies drop to 13.9 and $3.1 \%$ for $\beta=10^{-2}$ and $\beta=10^{-3}$, respectively.

\subsection{IC-59 final cut optimization}

To optimize the sensitivity for bright monopoles a Multivariate Analysis is used. It classifies each event by a Boosted Decision Trees (BDT) score in the range $[-1,+1][59,60]$. A BDT score of -1 characterizes a background-like event whereas a BDT score of +1 characterizes a signal-like event.

For the analysis, a sample of $10 \%$ of all experimental data (burn sample) was divided into two equally sized sets. BDTs have been trained on each combination $\left(\beta, \lambda_{\text {cat }}\right)$ of the signal Monte Carlo and on $50 \%$ of the corresponding burn sample, using combinations of the variables described above. The sensitivity was estimated from the other $50 \%$ of the burn sample by fitting an exponential function to the tail of the BDT score distribution. Over a large range of the BDT scores, the fit describes the data rather well. Still, its extension into the signal region has no strict physical justification.

The final cut on the BDT scores for each combination of $\left(\beta, \lambda_{\text {cat }}\right)$ is obtained by using the Model Rejection Factor (MRF) method [56]. For the chosen high catalysis cross 

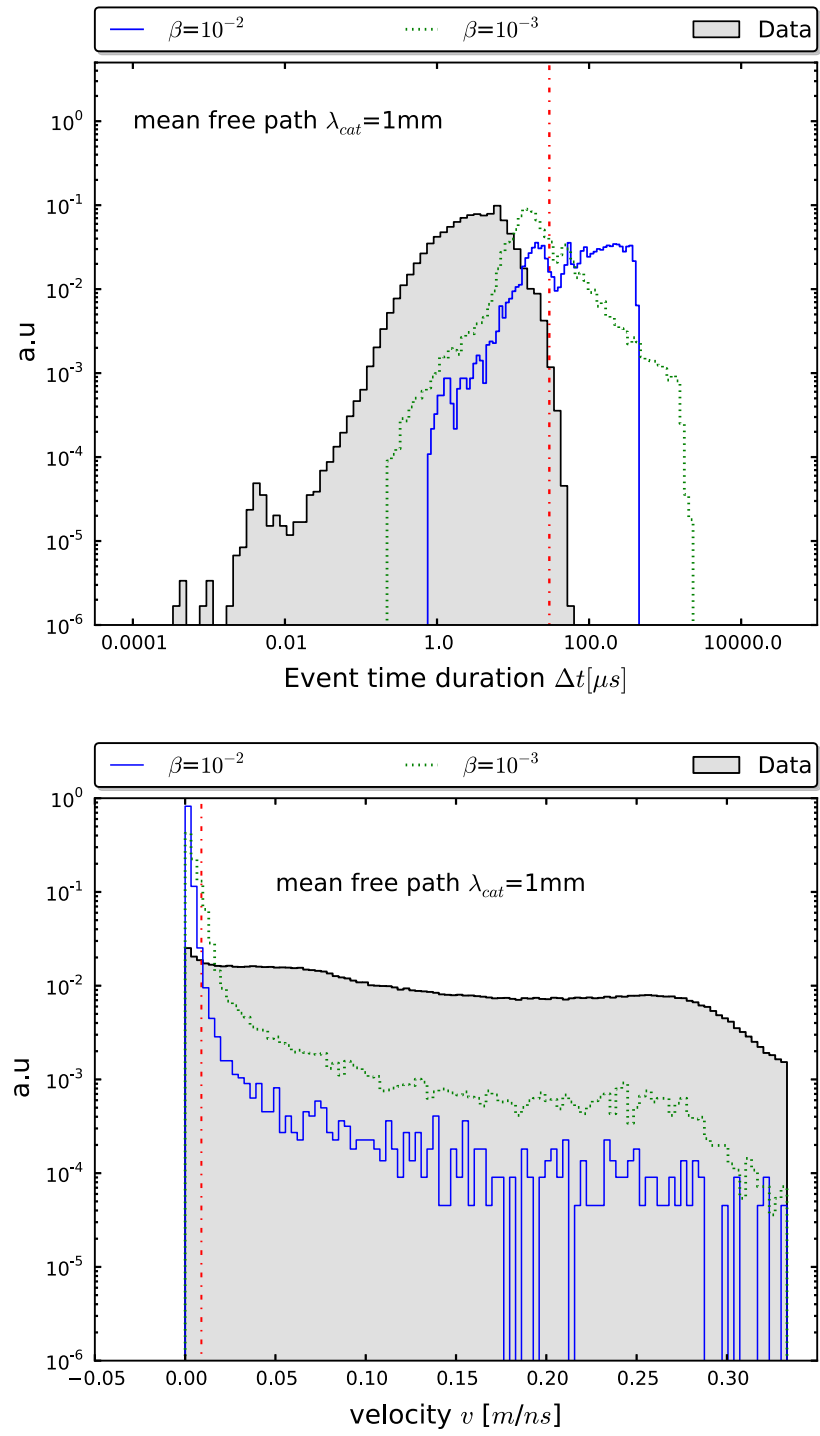

Fig. 16 Top Distribution of the event duration $\Delta \mathrm{t}$, for experimental data and simulated bright monopoles with $\lambda_{\text {cat }}=1 \mathrm{~mm}$ (i.e. $\sigma_{\mathrm{cat}}=$ $1.7 \cdot 10^{-22} \mathrm{~cm}^{2}$ ), before applying a cut. The green histogram represents monopoles with $\beta=10^{-3}$, the blue histogram with $\beta=10^{-2}$. The gray histogram represents the data. The red dashed line marks the value of the chosen cut which is set at $\Delta \mathrm{t}>30 \mu \mathrm{s}$. Bottom The same for the reconstructed speed $\mathrm{v}$ with a cut at $\mathrm{v}<9 \cdot 10^{-3} \mathrm{~m} / \mathrm{ns}$. Histograms are normalized to 1

sections the limits for three $\left(\beta, \lambda_{\text {cat }}\right)$ combinations are significantly better or comparable to those of the IC-86/DeepCore analysis. The fourth combination $\left(\beta=10^{-3}, \lambda_{\text {cat }}=1 \mathrm{~cm}\right)$ is not competitive because the optimal cut results in 42 expected background events for the full data sample.

\subsection{Results}

Figure 18 shows the BDT scores for data and signal $(\beta=$ $10^{-3}, \lambda_{\text {cat }}=1 \mathrm{~mm}$ ) for one year data taking (311.25 days live time) after the unblinding. The optimized cut on the BDT
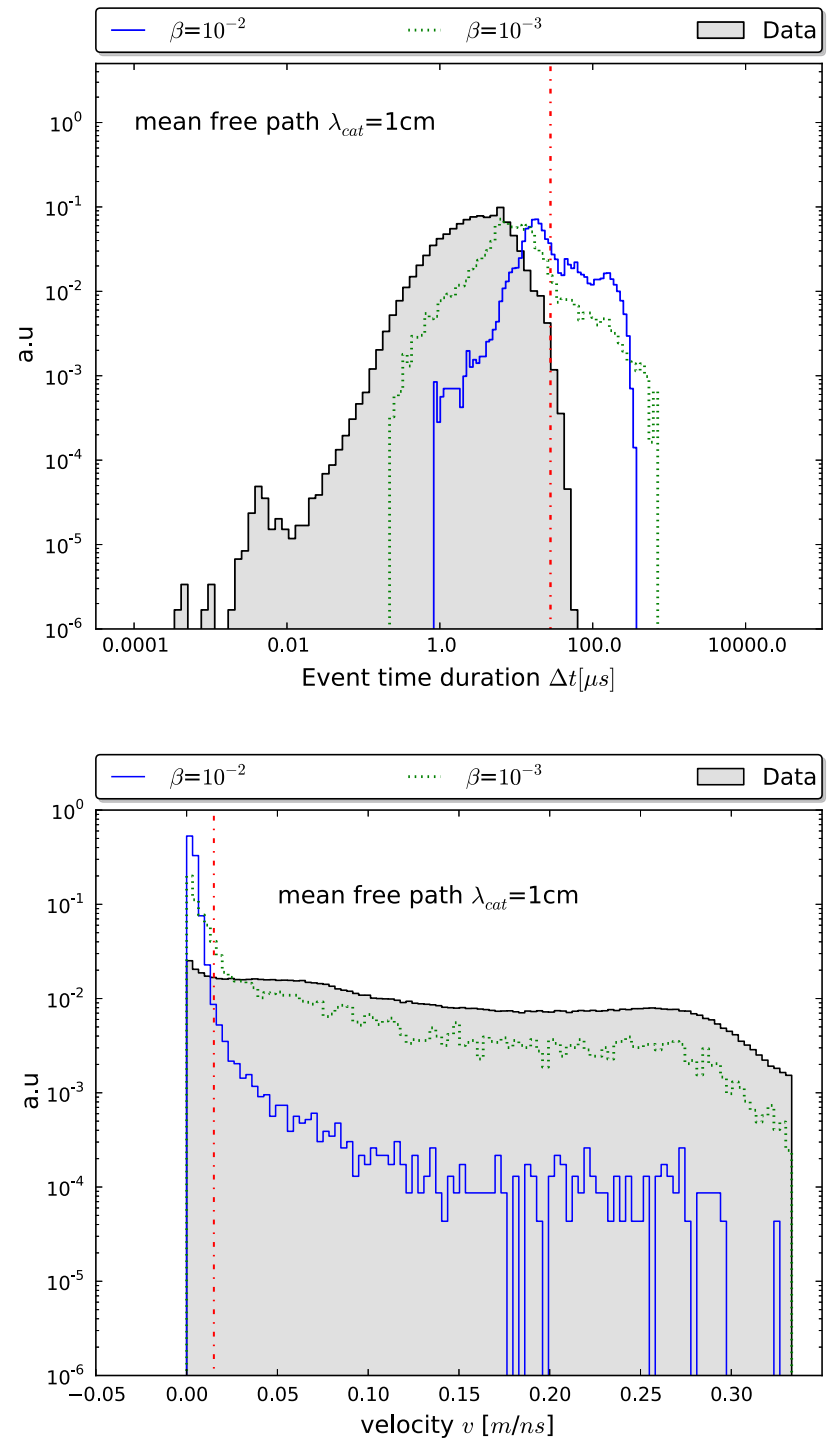

Fig. 17 Same as Fig. 16 but with $\lambda_{\text {cat }}=1 \mathrm{~cm}$, i.e. 10 times lower. The red dashed line marks the value of the chosen cut which is set at $\Delta \mathrm{t}>28 \mu \mathrm{s}$ for the event duration and $\mathrm{v}<1.5 \cdot 10^{-2} \mathrm{~m} / \mathrm{ns}$ for the speed. Histograms are normalized to 1

scores leaves only one event which merely passes the cut. No events pass the cuts for $\beta=10^{-2}, \lambda_{\text {cat }}=1 \mathrm{~mm}$ and $\beta=$ $10^{-2}, \lambda_{\text {cat }}=1 \mathrm{~cm}$. The one surviving event was inspected visually. It contains two nearly vertical high-energy muons which subsequently cross the whole detector and trigger two neighboring strings. It has a time duration $\Delta t=63.6 \mu \mathrm{s}$ and a reconstructed speed of $\mathrm{v}=8.5 \cdot 10^{-3} \mathrm{~m} / \mathrm{ns}$.

The expected number of background events after unblinding is calculated using the exponential fit to the BDT score distributions of the one year experimental data. The numbers of expected background events and of observed events, as well as the cut values on the BDT score for each parameter combination $\left(\beta, \lambda_{\text {cat }}\right)$ are shown in Table 3 . The higher number of expected background events compared to the actually 


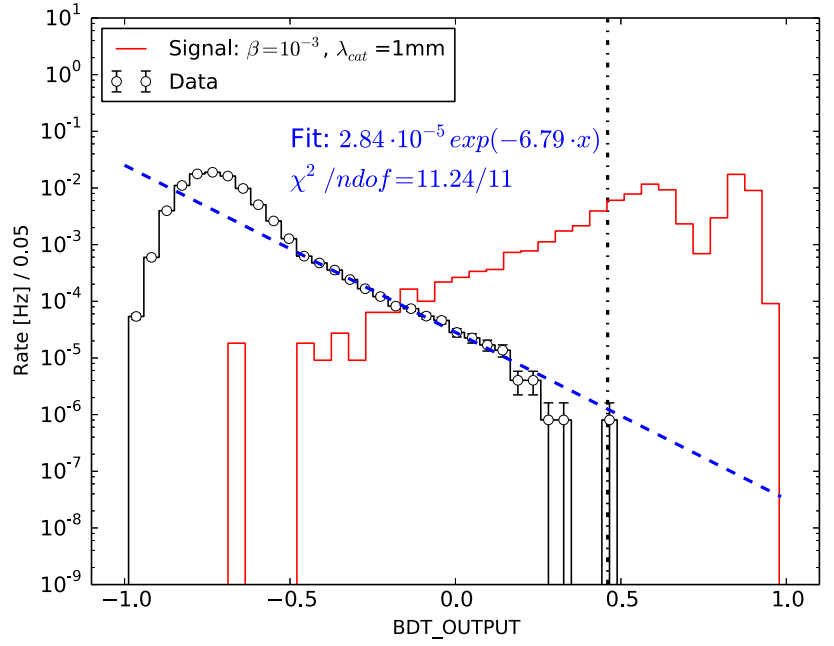

Fig. 18 Distribution of the BDT scores, after unblinding, for data and signal with $\lambda_{\text {cat }}=1 \mathrm{~mm}$, and speed $\beta=10^{-3}$. The dot dashed line shows the optimized cut on the BDT score obtained from the Model Rejection Factor method. One event survived the BDT cut and is compatible with the background

Table 3 Number of expected and observed events per year for every $\left(\beta, \lambda_{\text {cat }}\right)$ parameter combination. $N_{\text {expected }}$ is derived from the integral of the fitted BDT scores with an exponential. The integral ranges are from the BDT cut value to unity. The errors on the number of expected background event are $1 \sigma$ errors derived from a toy Monte Carlo experiment.

\begin{tabular}{|c|c|c|c|}
\hline & $\mathrm{BDT}_{\text {cut }}$ & $N_{\text {expected }}$ & $N_{\text {observed }}$ \\
\hline \multicolumn{4}{|c|}{$\sigma_{\text {cat }}=1.7 \cdot 10^{-22} \mathrm{~cm}^{2}, \lambda_{\text {cat }}=1 \mathrm{~mm}$} \\
\hline$\beta=10^{-2}$ & 0.46 & $0.6_{-0.1}^{+0.2}$ & 0 \\
\hline$\beta=10^{-3}$ & 0.48 & $4.8_{-0.6}^{+0.7}$ & 1 \\
\hline \multicolumn{4}{|c|}{$\sigma_{\text {cat }}=1.7 \cdot 10^{-23} \mathrm{~cm}^{2}, \lambda_{\text {cat }}=1 \mathrm{~cm}$} \\
\hline$\beta=10^{-2}$ & 0.5 & $3.0_{-0.5}^{+0.6}$ & 0 \\
\hline$\beta=10^{-3}$ & Not sensitive & Not sensitive & Not sensitive \\
\hline
\end{tabular}

observed number of events suggests that the exponential fit over-estimates the background rather than under-estimates it.

\section{Systematic uncertainties}

The calculation of upper flux limits takes into account the statistical and systematic uncertainties in the background and signal predictions. Because the number of expected background events is estimated from experimental data, only the statistical uncertainties of the fit parameters of the background model are relevant.

For signal the imperfect detector description is taken into account. For example in case of the IC-86/DeepCore search, the random noise leads to an increase of $n$-triplet for signal events. Furthermore the optical light detection efficiency is important. This efficiency takes into account the cumulative effect of the light yield of nucleon decays, where a single electromagnetic cascade is simulated instead of several daughter particles, the light propagation through the ice and its detection by the DOMs. These effects result in an uncertainty of the detection efficiency for magnetic monopoles which is used to derive the upper limits.

The impact of these uncertainties on the flux limits is estimated by simulating monopoles with simulation parameters changed within their estimated uncertainties. The uncertainties of the superimposed background noise, the light yield of nucleon decays and the light propagation through ice are estimated by their differences in the detection efficiencies of signal simulations taking into account different approaches (Sect. 2.3). For the IC-86/DeepCore analysis the superimposed noise can be described by random and correlated noise hits from experimental data or noise simulated as a Poisson process and atmospheric muons simulated using the software package CORSIKA. Since for the IC-59 analysis no unbiased experimental data exists the background noise can be simulated by a noise generator that also takes into account correlated noise hits. For reasons of simplification the proton decay is simulated as a single electromagnetic cascade with an isotropic direction which is valid as long as the mean free path is much smaller than the IceCube spacing. Due to kinematics in the proton decay (Eq. 3) two back-to-back electromagnetic cascades with an isotropic direction have to be simulated. The uncertainties due to this simplification are estimated by the differences between both approaches. For the light propagation through ice the two ice models described in $[49,50]$ are used. The uncertainty of the optical efficiency of DOMs can be estimated as $\pm 10 \%$. Signal simulations based on optical DOM efficiencies varied by $\pm 10 \%$ are compared with simulations based on the default settings. The differences in the detection efficiencies are used as an estimate for the uncertainty.

We quantify each systematic effect $i$ by the ratio of the resulting detection efficiency $\varepsilon_{i}$ relative to the detection efficiency with baseline assumptions $\varepsilon_{0}$ :

$R_{\mathrm{i}}\left(\beta, \sigma_{\mathrm{cat}}\right)=\frac{\varepsilon_{\mathrm{i}}\left(\beta, \sigma_{\mathrm{cat}}\right)}{\varepsilon_{0}\left(\beta, \sigma_{\mathrm{cat}}\right)}$.

The resulting changes are displayed in Table 4 .

Note that these calculations are limited by computing resources and correspondingly by the statistics of simulated events. The resulting statistical uncertainties of effective areas are typically a few percent as shown in Table 5 and included in the total error.

For the calculation of the final flux limits we perform high statistics computer experiments. In each we randomize the effect of each systematics effect $R_{\mathrm{i}}$ according to its specific uncertainty. For each parameter combination $\beta$ and $\sigma_{\text {cat }}$ this 
Table 4 The impact of different systematic uncertainties on the detection efficiencies of magnetic monopoles depending on the mean free path $\lambda_{\text {cat }}$ and the monopole speed $\beta$. The first column shows the impact of different assumptions for the superimposed background noise. Also the uncertainties of the simplified nucleon decay simulation (second column), the optical DOM efficiency (third column) and the optical ice properties (fourth column) are shown

\begin{tabular}{|c|c|c|c|c|c|c|c|c|}
\hline \multirow[t]{2}{*}{$\lambda_{\text {cat }}(\mathrm{m})$} & \multicolumn{2}{|c|}{ Noise simulation } & \multicolumn{2}{|c|}{ Nucleon decay simulation } & \multicolumn{2}{|c|}{ Optical DOM efficiency } & \multicolumn{2}{|c|}{ Optical ice properties } \\
\hline & $\beta=10^{-2}$ & $\beta=10^{-3}$ & $\beta=10^{-2}$ & $\beta=10^{-3}$ & $\beta=10^{-2}$ & $\beta=10^{-3}$ & $\beta=10^{-2}$ & $\beta=10^{-3}$ \\
\hline \multicolumn{9}{|l|}{ IC-86 } \\
\hline 3.0 & $+29 \% /-2 \%$ & $+23 \%$ & $+36 \%$ & $-16 \%$ & $+36 \% /-23 \%$ & $+46 \% /-29 \%$ & $+20 \% /-11 \%$ & $+72 \%$ \\
\hline 1.0 & $+52 \%$ & $+6 \% /-11 \%$ & $+27 \%$ & $+1 \% /-11 \%$ & $+14 \% /-7 \%$ & $+16 \% /-20 \%$ & $-17 \%$ & $+12 \%$ \\
\hline 0.3 & $+31 \%$ & $+5 \% /-8 \%$ & $+15 \%$ & $+1 \% /-8 \%$ & $\pm 10 \%$ & $\pm 11 \%$ & $-16 \%$ & $+8 \% /-2 \%$ \\
\hline 0.1 & $+19 \% /-1 \%$ & $+3 \% /-4 \%$ & $\pm 7 \%$ & $+1 \% /-6 \%$ & $+5 \% /-11 \%$ & $+8 \% /-6 \%$ & $-15 \%$ & $+1 \% /-6 \%$ \\
\hline 0.03 & $+17 \% /-2 \%$ & $+1 \% /-5 \%$ & $+9 \% /-4 \%$ & $+1 \% /-4 \%$ & $+10 \% /-5 \%$ & $+6 \% /-3 \%$ & $-9 \%$ & $-7 \%$ \\
\hline 0.01 & $+15 \% /-4 \%$ & $-4 \%$ & $+11 \% /-2 \%$ & $\pm 2 \%$ & $+12 \% /-1 \%$ & $+5 \% /-0.3 \%$ & $\pm 5 \%$ & $-10 \%$ \\
\hline 0.001 & $+15 \% /-4 \%$ & $-4 \%$ & $+11 \% /-2 \%$ & $\pm 2 \%$ & $+12 \% /-1 \%$ & $+5 \% /-0.3 \%$ & $\pm 5 \%$ & $-10 \%$ \\
\hline \multicolumn{9}{|l|}{ IC-59 } \\
\hline 0.01 & $+2 \%$ & - & $-5 \%$ & - & $+9 \% /-6 \%$ & - & $+7 \%$ & - \\
\hline 0.001 & $-3 \%$ & $+5 \%$ & $-3 \%$ & $-1 \%$ & $-5 \%$ & $+9 \% /-2 \%$ & $+2 \%$ & $+4 \%$ \\
\hline
\end{tabular}

Table 5 The statistical uncertainties of the calculated effective areas for different mean free path $\lambda_{\text {cat }}$ and speed $\beta$ for the IC-86 and IC-59 analyses

\begin{tabular}{llll}
\hline & $\lambda_{\text {cat }}(\mathrm{m})$ & \multicolumn{2}{l}{ Statistical uncertainties } \\
\cline { 3 - 4 } & & $\beta=10^{-2}(\%)$ & $\beta=10^{-3}(\%)$ \\
\hline IC-86 & 3.0 & 9 & 10 \\
& 1.0 & 8 & 5 \\
& 0.3 & 8 & 2 \\
& 0.1 & 8 & 2 \\
& 0.03 & 9 & 2 \\
& 0.01 & 10 & 2 \\
& 0.001 & 12 & 2 \\
IC-59 & 0.01 & 0.2 & - \\
& 0.001 & 0.1 & 0.1 \\
\hline
\end{tabular}

results in the effective probability density distribution for the relative change of the detection efficiency $R$ taking into account all uncertainties.

An example is shown in Fig. 19. Multiplying these distributions by the detection efficiency with baseline assumptions $\epsilon_{0}$ one gets the probability density distributions for the detection efficiencies.

\section{Flux limits}

The flux limits on non-relativistic magnetic monopoles are calculated assuming an isotropic flux and the proton decay channel $p \rightarrow \mathrm{e}^{+} \pi^{0}$ (Eq. 3) with the catalysis cross section $\sigma_{\text {cat }}$, which depends on the speed $\beta$ (Eq. 4). Using the quan-

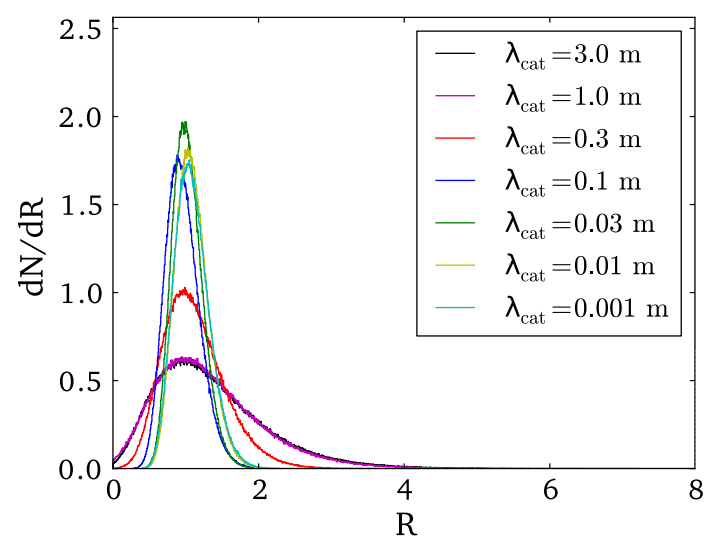

Fig. 19 Probability density distribution for systematic signal uncertainties for $\beta=10^{-2}$ monopoles for the IC-86/DeepCore analysis

tity $\hat{l}$ (Eq. 6) the flux limits can also be expressed without assuming a specific decay channel.

The flux limits are calculated based on a generalization of the approach by Rolke et al. [61], which takes into account the uncertainties of the signal detection efficiency and the expected number of background events. Therefore, a threedimensional likelihood fit is performed with the following parameters: expected number of signal events $\mu$, signal detection efficiency $\varepsilon$, and expected number of background events $n_{\mathrm{b}}$. The likelihood function is defined by

$L\left(\mu, \varepsilon, n_{\mathrm{b}} \mid n_{\mathrm{obs}}\right)=\frac{\lambda^{n_{\mathrm{obs}}} \cdot \mathrm{e}^{-\lambda}}{n_{\mathrm{obs}} !} \cdot f_{\mathrm{s}}(\varepsilon) \cdot f_{\mathrm{b}}\left(n_{\mathrm{b}}\right)$,

where the number of observed events $n_{\text {obs }}$ follows a Poisson distribution with the expectation value $\lambda=\varepsilon \mu+n_{\mathrm{b}}$. The functions $f_{\mathrm{s}}(\varepsilon)$ and $f_{\mathrm{b}}\left(n_{\mathrm{b}}\right)$ represent the probability den- 


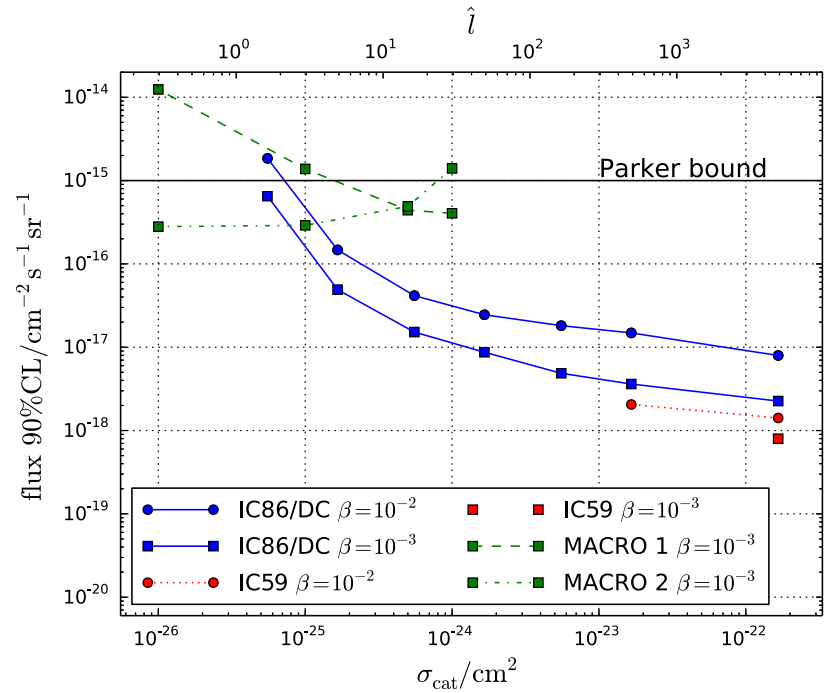

Fig. 20 Upper limits on the flux of non-relativistic magnetic monopoles depending on the speed $\beta$ and catalysis cross section $\sigma_{\text {cat }}$ of the IC-59 analysis and IC-86/DeepCore analysis. The dashed lines are limits published by the MACRO experiment [16]. Here, MACRO 1 is an analysis developed for monopoles catalyzing the proton decay. MACRO 2 is the standard MACRO-analysis, which is sensitive to monopoles ionizing the surrounding matter. Additionally, the IceCube limits are shown as a function of $\hat{l}$, which is proportional to the averaged Cherenkov photon yield per nucleon decay (not valid for MACRO limits)

sity distributions of the signal detection efficiency and the expected number of background events (Figs. 15 and 19).

The flux limits for each monopole speed $\beta$ and catalysis cross section $\sigma_{\text {cat }}$ are calculated by

$\Phi_{90}\left(\beta, \sigma_{\text {cat }}\right)=\frac{\mu_{90}}{A_{\text {gen }} \cdot t \cdot \Omega}$,

where $\mu_{90}$ is the upper limit at the $90 \%$ confidence level for $n_{\mathrm{obs}}=1$ event. The upper limit is obtained from the profile likelihood function defined in [61]. In order to exclude unphysical values of $\mu_{90}$ the expected number of signal events $\mu$ is constrained to be greater than or equal to zero. As a consequence of this method the upper limits at the $90 \%$ confidence level have a slight over-coverage of about $5 \%$. The other parameters are the size of the signal generation disc $A_{\text {gen }}$, the solid angle $\Omega=4 \pi \mathrm{sr}$ and the live time $t$.

Figure 20 shows the resulting direct detection limits on the flux of non-relativistic magnetic monopoles in comparison to the current best experimental flux limits by the MACRO experiment $[15,16]$.

Above $\sigma_{\text {cat }}=10^{-25} \mathrm{~cm}^{2}$ corresponding to $\lambda_{\text {cat }}<3 \mathrm{~m}$ the previous flux limits are improved by more than one order of magnitude. Moreover, for such large cross sections the monopole flux can be constrained up to a level

$\Phi_{90} \leq 10^{-18} \mathrm{~cm}^{-2} \mathrm{~s}^{-1} \mathrm{sr}^{-1}$,

which is three orders of magnitude below the Parker bound.
Assuming monopoles are the dominant part of Dark Matter, i.e. the relic mass density of monopoles is similar to the Dark Matter mass density, our most stringent flux limits constrain the monopole mass to be at least of the order of the Planck mass $m_{\mathrm{pl}}=1.22 \cdot 10^{19} \mathrm{GeV}$ [62]. This implies that monopoles with masses significantly smaller than the Planck mass do not contribute dominantly to the Dark Matter mass density.

Indirect searches for monopole induced proton decays set very strong bounds on monopoles with non-relativistic speeds, e.g. the limits from Super-Kamiokande [63] assuming gravitational trapping of monopoles in the sun. Also a variety of bounds based on observations of neutron stars, white dwarfs, and gas giants have been obtained. These bounds range from $\sim 10^{-18}-10^{-29} \mathrm{~cm}^{-2} \mathrm{~s}^{-1} \mathrm{sr}^{-1}$ and depend on the catalysis cross sections as well as on details of the assumed astrophysical scenarios [64-66]. Although the direct IceCube searches are not as stringent as indirect searches the former are not affected by astrophysical uncertainties. Thus the direct IceCube limits can be considered as a robust upper bound on the monopole flux, if the RubakovCallan effect is realized in nature.

\section{Summary and outlook}

Data taken from May 2011 until May 2012 with a dedicated slow-particle trigger and for the brightest monopoles data taken from May 2009 until May 2010 with standard IceCube triggers were analyzed. The analysis, which is based on data of the slow-particle trigger, was developed by using simulated monopole events and experimental data to estimate the background properties. For this first analysis of such a signal in IceCube a robust approach based on a single final selection criterion and the comparison between the number of expected background events and observed experimental events is chosen. Using the experimental data, the number of expected background events can be estimated to $n_{\mathrm{b}}=3.2_{-1.1}^{+1.8}$.

The IC-59 analysis based on standard IceCube triggers is sensitive only for bright monopoles with $\sigma_{\text {cat }}>1.7$. $10^{-23} \mathrm{~cm}^{2}$. The analysis used Boosted Decision Trees (BDT) to discriminate between monopole signal and background. The expected number of background events is derived from a fit of the BDT scores tails with an exponential function for each $\left(\beta, \lambda_{\text {cat }}\right)$. The number of observed events after unblinding is 1 for an expected background of $4.8_{-0.6}^{+0.7}$. This event contains multiple coincident muons, which renders it compatible with a background event. The obtained flux limits for $\beta=10^{-2}$ and $\lambda_{\text {cat }}=0.01 \mathrm{~m}, 0.001 \mathrm{~m}$ from the IC-59 analysis are better than the ones from the IC-86/DeepCore analysis because of the bigger effective area. For $\beta=10^{-3}$ the limits are comparable since the standard IceCube triggers 
are less sensitive to the monopole signal in comparison to the dedicated slow-particle trigger.

In both analyses no monopole signal has been observed. Thus, the limits on the flux of non-relativistic magnetic monopoles - catalyzing the proton decay-are improved by about more than one order of magnitude in comparison to MACRO [16] for most of the investigated parameter space and reach down to about three orders of magnitude below the Parker limit.

Since May 2012 the dedicated slow-particle trigger has been updated to the full IceCube detector. From this upgrade, we expect an improvement in sensitivity by roughly an order of magnitude [67]. This gain is supplemented by improvements of the data selection which have been developed after completion of this analysis. Examples are the implementation of a Kalman-filter-based HLC hit selection, which improves the angular and speed reconstruction, and the implementation of an event selection based on a Boosted Decision Tree [68].

Acknowledgments We acknowledge the support from the following agencies: U.S. National Science Foundation-Office of Polar Programs, U.S. National Science Foundation-Physics Division, University of Wisconsin Alumni Research Foundation, the Grid Laboratory Of Wisconsin (GLOW) grid infrastructure at the University of Wisconsin - Madison, the Open Science Grid (OSG) grid infrastructure; U.S. Department of Energy, and National Energy Research Scientific Computing Center, the Louisiana Optical Network Initiative (LONI) grid computing resources; Natural Sciences and Engineering Research Council of Canada, WestGrid and Compute/Calcul Canada; Swedish Research Council, Swedish Polar Research Secretariat, Swedish National Infrastructure for Computing (SNIC), and Knut and Alice Wallenberg Foundation, Sweden; German Ministry for Education and Research (BMBF), Deutsche Forschungsgemeinschaft (DFG), L.B. was funded by the DFG Sonderforschungsbereich 676, Helmholtz Alliance for Astroparticle Physics (HAP), Research Department of Plasmas with Complex Interactions (Bochum), Germany; Fund for Scientific Research (FNRS-FWO), FWO Odysseus programme, Flanders Institute to encourage scientific and technological research in industry (IWT), Belgian Federal Science Policy Office (Belspo); University of Oxford, United Kingdom; Marsden Fund, New Zealand; Australian Research Council; Japan Society for Promotion of Science (JSPS); the Swiss National Science Foundation (SNSF), Switzerland; National Research Foundation of Korea (NRF); Danish National Research Foundation, Denmark (DNRF).

Open Access This article is distributed under the terms of the Creative Commons Attribution License which permits any use, distribution, and reproduction in any medium, provided the original author(s) and the source are credited.

Funded by $\mathrm{SCOAP}^{3}$ / License Version CC BY 4.0.

\section{References}

1. P.A. Dirac, Proc. R. Soc. Lond. A 133, 60 (1931)

2. H. Georgi, S. Glashow, Phys. Rev. Lett. 32, 438 (1974)

3. G. 't Hooft, Nucl. Phys. B 79, 276 (1974)

4. A.M. Polyakov, JETP Lett. 20, 194 (1974)

5. H. Georgi, H.R. Quinn, S. Weinberg, Phys. Rev. Lett. 33, 451 (1974)
6. M. Daniel, G. Lazarides, Q. Shafi, Nucl. Phys. B 170, 156 (1980)

7. G. Lazarides, C. Panagiotakopoulos, Q. Shafi, Phys. Rev. Lett. 58, 1707 (1987)

8. T.W. Kephart, Q. Shafi, Phys. Lett. B 520, 313 (2001). hep-ph/0105237

9. S.D. Wick, T.W. Kephart, T.J. Weiler, P.L. Biermann, Astropart. Phys. 18, 663 (2003). astro-ph/0001233

10. T. Kibble, J. Phys. A 9, 1387 (1976)

11. J. Preskill, Ann. Rev. Nucl. Part. Sci. 34, 461 (1984)

12. M.S. Turner, E.N. Parker, T. Bogdan, Phys. Rev. D 26, 1296 (1982)

13. D.E. Groom, Phys. Rep. 140, 323 (1986)

14. F.C. Adams et al., Phys. Rev. Lett. 70, 2511 (1993)

15. MACRO Collaboration, M. Ambrosio et al., Eur. Phys. J. C 25, 511 (2002). hep-ex/0207020

16. MACRO Collaboration, M. Ambrosio et al., Eur. Phys. J. C 26, 163 (2002). hep-ex/0207024

17. IceCube Collaboration, R. Abbasi et al., Phys. Rev. D 87, 022001 (2013). arXiv:1208.4861

18. IceCube Collaboration, M. Aartsen et al., p. 9 (2013). arXiv: 1309.7007

19. IceCube Collaboration, J. Ahrens et al . Astropart. Phys. 20, 507 (2004). astro-ph/0305196

20. D.R. Tompkins, Phys. Rev. 138, B248 (1965)

21. IceCube Collaboration, R. Abbasi et al., Nucl. Instrum. Meth. A618, 139 (2010). arXiv:1002.2442

22. IceCube Collaboration, R. Abbasi et al., Nucl. Instrum. Meth. A601, 294 (2009). arXiv:0810.4930

23. IceCube Collaboration, A. Achterberg et al., Astropart. Phys. 26, 155 (2006). astro-ph/0604450

24. IceCube Collaboration, R. Abbasi et al., Astropart. Phys. 35, 615 (2012). arXiv: 1109.6096

25. S. Ahlen, Rev. Mod. Phys. 52, 121 (1980)

26. Y. Kazama, C.N. Yang, A.S. Goldhaber, Phys. Rev. D 15, 2287 (1977)

27. F. Bloch, Zeitschrift für Physik A Hadrons Nuclei 81, 363 (1933)

28. S. Ahlen, K. Kinoshita, Phys. Rev. D 26, 2347 (1982)

29. D. Ritson, SLAC-PUB-2950 (1982)

30. V. Rubakov, Nucl. Phys. B 203, 311 (1982)

31. J. Callan, Curtis G., Nucl. Phys. B 212, 391 (1983)

32. S. Dawson, A.N. Schellekens, Phys. Rev. D 27, 2119 (1983)

33. T. Walsh, P. Weisz, T.T. Wu, Nucl. Phys. B 232, 349 (1984)

34. S. Dawson, A.N. Schellekens, Phys. Rev. D 28, 3125 (1983)

35. V. Rubakov, M. Serebryakov, Nucl. Phys. B 237, 329 (1984)

36. V. Rubakov, Rept. Prog. Phys. 51, 189 (1988)

37. J. Arafune, M. Fukugita, Phys. Rev. Lett. 50, 1901 (1983)

38. P. Nath, P. Fileviez Perez, Phys. Rep. 441, 191 (2007). hep-ph/0601023

39. M. Kowalski, Search for neutrino-induced cascades with the AMANDA-II detector. PhD thesis, HU-Berlin, 2004. http://edoc. hu-berlin.de/dissertationen/kowalski-marek-paul-2004-01-13/ PDF/Kowalski

40. L. Radel, C. Wiebusch, Astropart. Phys. 38, 53 (2012). arXiv: 1206.5530

41. R. Becker-Szendy et al., Phys. Rev. D 49, 2169 (1994)

42. M. Fukugita, A. Suzuki, Physics and Astrophysics of Neutrinos (Springer, Berlin, 1994)

43. G. Domogatsky et al., Present Status of Baikal Deep Underwater Experiment, pp. 737-745 (1986)

44. BAIKAL Collaboration, L.B. Bezrukov et al. (1995) astro-ph/9601160

45. BAIKAL Collaboration, I. Belolaptikov et al., Astropart. Phys. 7, 263 (1997)

46. A. Pohl, Search for subrelativistic particles with the AMANDA neutrino telescope. PhD thesis (2009). http://wwwiexp.desy.de/ groups/astroparticle/pubs/ Thesis. Arvid. 090210

47. J. Derkaoui et al., Astropart. Phys. 9, 173 (1998) 
48. J. Lundberg et al., Nucl. Instrum. Meth. A 581, 619 (2007). astro-ph/0702108

49. M. Ackermann et al., J. Geophys. Res. D 111, 13203 (2006)

50. IceCube Collaboration, M. Aartsen et al., Nucl. Instrum. Meth. A 711, 73 (2013). arXiv:1301.5361

51. J. Capdevielle et al., The Karlsruhe Extensive Air Shower Simulation Code CORSIKA KfK (Series) (Kernforschungszentrum, Institut für Kernphysik, 1992)

52. R. Fletcher, T. Gaisser, P. Lipari, T. Stanev, Phys. Rev. D 50, 5710 (1994)

53. J.R. Hoerandel, Astropart. Phys. 19, 193 (2003). astro-ph/0210453

54. T. Glüsenkamp, On the Detection of Subrelativistic Magnetic Monopoles with the Ice Cube Neutrino Observatory (Diplomarbeit, RWTH Aachen, 2010)

55. AMANDA Collaboration, J. Ahrens et al., Nucl. Instrum. Meth. A 524, 169 (2004). astro-ph/0407044

56. G.C. Hill, K. Rawlins, Astropart. Phys. 19, 393 (2003). astro-ph/0209350

57. IceCube Collaboration, M. Aartsen et al. (2013). arXiv:1307.6669

58. IceCube Collaboration, M. Aartsen et al. (2013). arXiv:1312.0104

59. A. Hocker et al., PoS ACAT, 040 (2007). physics/0703039
60. P. Byron, H. Yang, J. Zhub, Boosted decision trees, a powerful event classifier. In Statistical Problems in Particle Physics, Astrophysics and Cosmology: Proceedings of PHYSTAT05, vol. 40, p. 139. Imperial College Press, London (2006)

61. W.A. Rolke, A.M. Lopez, J. Conrad, Nucl. Instrum. Meth. A551, 493 (2005). physics/0403059

62. E. Kolb, M. Turner, The Early Universe, Frontiers in Physics (Addison-Wesley Longman, Incorporated, 1990)

63. Super-Kamiokande Collaboration, K. Ueno et al., Astropart. Phys. 36, 131 (2012). arXiv: 1203.0940

64. J.A. Harvey, Nucl. Phys. B 236, 255 (1984)

65. K. Freese, E. Krasteva, Phys. Rev. D 59, 063007 (1999). astro-ph/9804148

66. J. Arafune, M. Fukugita, S. Yanagita, Phys. Rev. D 32, 2586 (1985)

67. IceCube Collaboration, M. Aartsen et al., p. 25 (2013). arXiv: 1309.7007

68. S. Zierke, Verbesserung von Rekonstruktions- und Datenselektions-Methoden für die Messung subrelativistischer magnetischer Monopole mit IceCube. Master thesis, RWTH Aachen (2013) 